$X X X, p p .1-20$

Advance Access publication on XXX

\title{
High-dimensional empirical likelihood inference
}

\author{
BY JINYUAN CHANG \\ School of Statistics and Institute of Big Data, Southwestern University of Finance and \\ Economics, Chengdu, Sichuan 611130, China \\ changjinyuan@swufe.edu.cn \\ SONG XI CHEN \\ Guanghua School of Management, Peking University, Beijing 100871, China \\ csx@gsm.pku.edu.cn \\ CHENG YONG TANG \\ Department of Statistical Science, Temple University, Philadelphia, Pennsylvania 19122, U.S.A. \\ yongtang@temple.edu \\ AND TONG TONG WU \\ Department of Biostatistics and Computational Biology, University of Rochester, Rochester, \\ New York 14642, U.S.A. \\ Tongtong_Wu@urmc.rochester.edu
}

\section{SUMMARY}

High-dimensional statistical inference with general estimating equations are challenging and remain less explored. In this paper, we study two problems in the area: confidence set estimation for multiple components of the model parameters, and model specifications test. For the first one, we propose to construct a new set of estimating equations such that the impact from estimating the high-dimensional nuisance parameters becomes asymptotically negligible. The new construction enables us to estimate a valid confidence region by empirical likelihood ratio. For the second one, we propose a test statistic as the maximum of the marginal empirical likelihood ratios to quantify data evidence against the model specification. Our theory establishes the validity of the proposed empirical likelihood approaches, accommodating over-identification and exponentially growing data dimensionality. The numerical studies demonstrate promising performance and potential practical benefits of the new methods.

Some key words: Empirical likelihood, General estimating equations, High-dimensional statistical inferences, Nuisance parameter, Over-identification.

\section{INTRODUCTION}

General estimating equations are broadly applicable for solving statistical inference problems, and they commonly involve over-identification: a general situation where the number of restrictions is larger than that of the model parameters. Such a feature is advantageous. As a distinguished example, the generalized method of moments (Hansen, 1982) allows incorporating a flexible number of moment conditions in model building and subsequent statistical inferences; 
see also Hansen \& Singleton (1982). Empirical likelihood (Owen, 2001), coupled with general estimating equations, has been demonstrated powerful for statistical inference since the seminal work of Qin \& Lawless (1994). Without requiring to specify a full parametric probability distribution, empirical likelihood conveniently supports statistical inference with many desirable features including the Wilks' type theorems, data adaptive yet shape constraint-free confidence regions, and flexibility in combining multiple sources of data information.

Recently, there has been a surge in research for high-dimensional statistical problems. A class of approaches are facilitated by sparse model parameters whose many components are zeros. Penalized likelihood approaches have been demonstrated effective for estimating sparse model parameters; see the overview by Fan \& Lv (2010), the monographs Bühlmann \& van de Geer (2011), Hastie et al. (2015), and references therein. Nevertheless, most existing penalized likelihood methods are constructed from conventional tools such as the least squares criterion, and the log-likelihood functions. Hence, they do not accommodate problems with general estimating equations, leaving this influential device less utilized.

High-dimensionality is challenging for empirical likelihood; see Hjort et al. (2009) and Chen et al. (2009). Facilitated by empirical likelihood, Leng \& Tang (2012) and Chang et al. (2015) consider penalized empirical likelihood with general estimating equations and show that sparse estimator and statistical inferential procedures with good properties are achievable. However, those results only hold when the numbers of estimating equations and model parameters diverge at some slow polynomial rate of the sample size. Recently, Chang et al. (2018) introduce a new penalized empirical likelihood method that can accommodate exponentially growing numbers of estimating equations and model parameters. Their method effectively selects a subset of the estimating equations for estimating the nonzero components of the sparse model parameters. The study in Chang et al. (2018) only focuses on estimations and does not cover broader concerns such as testing hypotheses or constructing confidence regions.

We consider in this paper two inference problems with general estimating equations using empirical likelihood. To our best knowledge, this is the first attempt in the literature to accommodate over-identification in high-dimensional settings. In our presentation, we call a case as "low-dimensional" when it deals with either fixed or slowly diverging numbers of model parameters and estimating equations. The first problem is how to construct a confidence region for low-dimensional multiple components of the high-dimensional model parameters. Here the estimation error associated with the other components of the model parameters - so called nuisance parameters - is cumbersome. To overcome this difficulty, we propose to construct empirical likelihood with a new set of low-dimensional estimating equations for those specified components. By projecting the original estimating equations with a linear transformation matrix whose rows are asymptotically orthogonal to the column space of the gradient matrix with respect to the nuisance parameters, the impact due to the estimation of the nuisance parameters becomes asymptotically negligible. Under the new construction, a valid confidence region can be constructed using empirical likelihood ratio. The second problem is how to test whether or not a set of over-identified moment conditions are correctly specified. Our approach here is to calculate the marginal empirical likelihood ratios from a set of estimating functions evaluated at some consistent estimates. If a moment condition is mis-specified, the corresponding marginal empirical likelihood ratio will diverge. Therefore, we propose a novel high-dimensional over-identification test by assessing the maximum of the marginal empirical likelihood ratios.

Our investigation contributes to several areas. Foremost, it expands the scope and deepens the understanding of high-dimensional empirical likelihood methods. We show that by appropriately mapping, empirical likelihood still inherits the desirable merits for statistical inference with general estimating equations as in Qin \& Lawless (1994). The key is to handle the high-dimensional 
nuisance parameters - a problem of foundational importance in the empirical likelihood literature; see, among others, Lazar \& Mykland (1999), Chen \& Cui (2006, 2007), Hjort et al. (2009), and the recent investigation of Bravo et al. (2019). Our treatment using a linear transformation is new, and it provides a crucial device of its own interests when investigating empirical likelihood; see $\S 3 \cdot 1$ for details and discussions. Second, our empirical likelihood-based over-identification test offers a new specification assessment tool to check the moment conditions. In conventional cases, the validity of the moment conditions can be assessed by the famous Sargan-Hansen's $J$-test (Sargan, 1958; Hansen, 1982) and the empirical likelihood ratio test (Qin \& Lawless, 1994). Unfortunately, those testing procedures cannot be applied to high-dimensional problems because the test statistics are not even well defined when the number of estimating equations is larger than the sample size. For filling this gap, our method provides a suitable and viable solution in high-dimensional settings. Moreover, our approach is the first that can simultaneously handle multiple components of the model parameters and over-identification inference problems. To our knowledge, existing high-dimensional methods of confidence set estimation focus on univariate analyses with no over-identification; see Zhang \& Zhang (2013), van de Geer et al. (2014), Lee et al. (2016), Tibshirani et al. (2016) and Ning \& Liu (2017). In the context of estimating equations, a recent study in Neykov et al. (2018) estimates univariate confidence interval in highdimensional just-identified settings, i.e., the same number of model parameters and estimating equations. Obviously, our approach applies more broadly. Our real data analysis with a most recent longitudinal data set from the Trial of Activity for Adolescent Girls demonstrates that the empirical likelihood methods with over-identification can provide an opportunity for potentially more accurate statistical inference in practice.

\section{PRELIMINARIES}

Let $X_{1}, \ldots, X_{n}$ be $d$-dimensional independently and identically distributed observations, and $\theta=\left(\theta_{1}, \ldots, \theta_{p}\right)^{\mathrm{T}} \in \Theta$ be a $p$-dimensional model parameter. With an $r$-dimensional estimating function $g(X ; \theta)=\left\{g_{1}(X ; \theta), \ldots, g_{r}(X ; \theta)\right\}^{\mathrm{T}}$, a data model involving $\theta$ is specified by

$$
E\left\{g\left(X_{i} ; \theta_{0}\right)\right\}=0
$$

where $\theta_{0}=\left(\theta_{0,1}, \ldots, \theta_{0, p}\right)^{\mathrm{T}} \in \Theta$ is the unknown truth. Here, one can view $\left\{g\left(X_{i} ; \theta\right)\right\}_{i=1}^{n}$ as a triangular array, where $r, d, p, X_{i}, \theta$ and $g(\cdot ; \cdot)$ may all depend on the sample size $n$.

For simplicity, when no confusion arises in the sequel, we use $h_{i}(\theta)$ as equivalent to $h\left(X_{i} ; \theta\right)$ for a generic $q$-dimensional function $h(\cdot ; \cdot)=\left\{h_{1}(\cdot ; \cdot), \ldots, h_{q}(\cdot ; \cdot)\right\}^{\mathrm{T}}$ and denote by $h_{i, k}(\theta)$ the $k$-th component of $h_{i}(\theta)$. Let $\bar{h}(\theta)=n^{-1} \sum_{i=1}^{n} h_{i}(\theta)$ and $h_{k}(\theta)=n^{-1} \sum_{i=1}^{n} h_{i, k}(\theta)$. For a given index set $\mathcal{L} \subset\{1, \ldots, q\}$, we denote by $h_{\mathcal{L}}(\cdot ; \cdot)$ the subvector of $h(\cdot ; \cdot)$ collecting the components indexed by $\mathcal{L}$. Analogously, let $h_{i, \mathcal{L}}(\theta)=h_{\mathcal{L}}\left(X_{i} ; \theta\right)$ and $\bar{h}_{\mathcal{L}}(\theta)=n^{-1} \sum_{i=1}^{n} h_{i, \mathcal{L}}(\theta)$. For a matrix $B=\left(b_{i, j}\right)_{s_{1} \times s_{2}}$, let $B^{\otimes 2}=B B^{\mathrm{T}},|B|_{\infty}=\max _{1 \leq i \leq s_{1}, 1 \leq j \leq s_{2}}\left|b_{i, j}\right|$, and $\|B\|_{s}$ denote the matrix $L_{s}$-operator norm of $B$. When $s_{2}=1,|B|_{s}$ denotes the vector $L_{s}$-norm of the $s_{1}$-dimensional vector $B$.

\subsection{Current development of high-dimensional empirical likelihood}

Since model estimation is the foundation of subsequent inference problems, let us start with an overview of the penalized empirical likelihood estimation approach in Chang et al. (2018):

$$
\hat{\theta}_{\mathrm{PEL}}=\arg \min _{\theta \in \Theta} \max _{\lambda \in \hat{\Lambda}_{n}(\theta)}\left[\sum_{i=1}^{n} \log \left\{1+\lambda^{\mathrm{T}} g_{i}(\theta)\right\}+n \sum_{k=1}^{p} P_{1, \pi}\left(\left|\theta_{k}\right|\right)-n \sum_{j=1}^{r} P_{2, \nu}\left(\left|\lambda_{j}\right|\right)\right],
$$


where $\lambda=\left(\lambda_{1}, \ldots, \lambda_{r}\right)^{\mathrm{T}}, \hat{\Lambda}_{n}(\theta)=\left\{\lambda \in \mathbb{R}^{r}: \lambda^{\mathrm{T}} g_{i}(\theta) \in \mathcal{U}\right.$ for any $\left.i=1, \ldots, n\right\}$ with an open interval $\mathcal{U}$ containing zero, and $P_{1, \pi}(\cdot)$ and $P_{2, \nu}(\cdot)$ are two penalty functions with tuning parameters $\pi$ and $\nu$, respectively. For any penalty function $P_{\tau}(\cdot)$ with tuning parameter $\tau$, let $\rho(t ; \tau)=\tau^{-1} P_{\tau}(t)$ for any $t \in[0, \infty)$ and $\tau \in(0, \infty)$. Define

$$
\begin{aligned}
\mathcal{P}=\left\{P_{\tau}(\cdot):\right. & \rho(t ; \tau) \text { is increasing in } t \in[0, \infty) \text { and has continuous } \\
& \text { derivative } \rho^{\prime}(t ; \tau) \text { for any } t \in(0, \infty) \text { with } \rho^{\prime}\left(0^{+} ; \tau\right) \in \\
& \left.(0, \infty), \text { where } \rho^{\prime}\left(0^{+} ; \tau\right) \text { is independent of } \tau\right\} .
\end{aligned}
$$

Such a class is broad and general, including the $L_{1}$ penalty, the smoothly clipped absolute deviation penalty (Fan \& Li, 2001), the minimax concave penalty (Zhang, 2010), and more. Let $\mathcal{S}=\left\{1 \leq k \leq p: \theta_{0, k} \neq 0\right\}$ with $|\mathcal{S}|=s \ll n$, i.e., the truth $\theta_{0}$ is sparse. From Chang et al. (2018), $\hat{\theta}_{\mathrm{PEL}}$ is consistent under some regularity conditions, stated in the following proposition.

Proposition 1. Let $P_{1, \pi}(\cdot), P_{2, \nu}(\cdot) \in \mathcal{P}$ and $P_{2, \nu}(\cdot)$ be convex with bounded second-order derivative around 0 . If Conditions A1-A6 and the restrictions (R.3) in the Appendix hold, there is a local minimizer $\hat{\theta}_{\mathrm{PEL}} \in \Theta$ in (2) such that (i) $\left|\hat{\theta}_{\mathrm{PEL}, \mathcal{S}}-\theta_{0, \mathcal{S}}\right|_{\infty}=O_{p}\left(\alpha_{n}\right)$ for some $\alpha_{n} \rightarrow 0$ as $n \rightarrow \infty$; and (ii) $\operatorname{pr}\left(\hat{\theta}_{\mathrm{PEL}, \mathcal{S}^{c}}=0\right) \rightarrow 1$ as $n \rightarrow \infty$.

From (R.3) in the Appendix, Proposition 1 holds even if $r$ and $p$ grow exponentially with $n$. On one hand, using a convex penalty $P_{2, \nu}(\cdot)$ makes the loss function concave with respect to $\lambda$, which leads to a unique maximizer $\lambda(\theta)$ in the inner optimization of (2) for each given $\theta$. On the other hand, due to the convexity of $P_{2, \nu}(\cdot)$, there exists an asymptotic bias of order slower than $n^{-1 / 2}$ in $\hat{\theta}_{\mathrm{PEL}, \mathcal{S}}$. We observe that regularizing $\lambda$ in (2) leads to a sparse solution $\hat{\lambda}$ corresponding to $\hat{\theta}_{\mathrm{PEL}}$, which effectively selects components in $g(\cdot ; \cdot)$. Write $\hat{\lambda}=\left(\hat{\lambda}_{1}, \ldots, \hat{\lambda}_{r}\right)^{\mathrm{T}}$ and $\mathcal{R}_{n}=\operatorname{supp}(\hat{\lambda})$. Similarly, denote $\rho_{2}(t ; \nu)=\nu^{-1} P_{2, \nu}(t)$ for any $t>0$ and $\hat{\eta}=\left(\hat{\eta}_{1}, \ldots, \hat{\eta}_{r}\right)^{\mathrm{T}}$ with $\hat{\eta}_{j}=\nu \rho_{2}^{\prime}\left(\left|\hat{\lambda}_{j}\right| ; \nu\right) \operatorname{sgn}\left(\hat{\lambda}_{j}\right)$ for $\hat{\lambda}_{j} \neq 0$ and $\hat{\eta}_{j} \in\left[-\nu \rho_{2}^{\prime}\left(0^{+}\right), \nu \rho_{2}^{\prime}\left(0^{+}\right)\right]$for $\hat{\lambda}_{j}=0$. Define $\hat{V}_{\mathcal{R}_{n}}\left(\hat{\theta}_{\mathrm{PEL}}\right)=n^{-1} \sum_{i=1}^{n} g_{i, \mathcal{R}_{n}}\left(\hat{\theta}_{\mathrm{PEL}}\right)^{\otimes 2}$ and $\hat{J}_{\mathcal{R}_{n}}=\left[\left\{\nabla_{\theta_{\mathcal{S}}} \bar{g}_{i, \mathcal{R}_{n}}\left(\hat{\theta}_{\mathrm{PEL}}\right)\right\}^{\mathrm{T}} \hat{V}_{\mathcal{R}_{n}}^{-1 / 2}\left(\hat{\theta}_{\mathrm{PEL}}\right)\right]^{\otimes 2}$. To achieve the best performance, the bias-corrected estimator is defined by

$$
\hat{\theta}_{\mathrm{PELbc}}=\hat{\theta}_{\mathrm{PEL}}-\hat{\psi}_{*}
$$

where the $p$-dimensional vector $\hat{\psi}_{*}$ satisfies $\hat{\psi}_{*, \mathcal{S}^{c}}=0$ and $\hat{\psi}_{*, \mathcal{S}}=\hat{\psi}$ with $s$-dimensional vector $\hat{\psi}=\hat{J}_{\mathcal{R}_{n}}^{-1}\left\{\nabla_{\theta_{\mathcal{S}}} \bar{g}_{\mathcal{R}_{n}}\left(\hat{\theta}_{\mathrm{PEL}}\right)\right\}^{\mathrm{T}} \hat{V}_{\mathcal{R}_{n}}^{-1}\left(\hat{\theta}_{\mathrm{PEL}}\right) \hat{\eta}_{\mathcal{R}_{n}}$. Let $V_{\mathcal{R}_{n}}\left(\theta_{0}\right)=E_{\mathcal{R}_{n}}\left\{g_{i, \mathcal{R}_{n}}\left(\theta_{0}\right)^{\otimes 2}\right\}$ and $J_{\mathcal{R}_{n}}=$ $\left\{\left[E_{\mathcal{R}_{n}}\left\{\nabla_{\theta_{\mathcal{S}}} g_{i, \mathcal{R}_{n}}\left(\theta_{0}\right)\right\}\right]^{\mathrm{T}} V_{\mathcal{R}_{n}}^{-1 / 2}\left(\theta_{0}\right)\right\}^{\otimes 2}$. Due to the randomness of the index set $\mathcal{R}_{n}$, we only take the expectation with respect to $X_{i}$ and treat $\mathcal{R}_{n}$ as given when we define $E_{\mathcal{R}_{n}}\left\{g_{i, \mathcal{R}_{n}}\left(\theta_{0}\right)^{\otimes 2}\right\}$ and $E_{\mathcal{R}_{n}}\left\{\nabla_{\theta_{\mathcal{S}}} g_{i, \mathcal{R}_{n}}\left(\theta_{0}\right)\right\}$. Properties of $\hat{\theta}_{\text {PELbc }}$ are summarized in the following proposition.

Proposition 2. Let $P_{1, \pi}(\cdot), P_{2, \nu}(\cdot) \in \mathcal{P}$ and $P_{2, \nu}(\cdot)$ be convex with bounded second-order derivative around 0 . If Conditions $\mathrm{A} 1-\mathrm{A} 8$ and the restrictions (R.4) in the Appendix hold, $\hat{\theta}_{\mathrm{PELbc}}$ in (4) satisfies: (i) $\hat{\theta}_{\mathrm{PELbc}, \mathcal{S}}-\theta_{0, \mathcal{S}}=-J_{\mathcal{R}_{n}}^{-1}\left[E_{\mathcal{R}_{n}}\left\{\nabla_{\theta_{\mathcal{S}}} g_{i, \mathcal{R}_{n}}\left(\theta_{0}\right)\right\}\right]^{\mathrm{T}} V_{\mathcal{R}_{n}}^{-1}\left(\theta_{0}\right) \bar{g}_{\mathcal{R}_{n}}\left(\theta_{0}\right)+\Delta_{n}$ with $\left|\Delta_{n}\right|_{2}=O_{p}\left(\phi_{n}\right)$ for some $\phi_{n}=o\left(n^{-1 / 2}\right)$; and (ii) $\operatorname{pr}\left(\hat{\theta}_{\mathrm{PELbc}, \mathcal{S}^{c}}=0\right) \rightarrow 1$ as $n \rightarrow \infty$.

To compute the bias-corrected estimator $\hat{\theta}_{\text {PELbc }}$, the support $\mathcal{S}$ of $\theta_{0}$ is needed. In practice, we may use the support of $\hat{\theta}_{\mathrm{PEL}}$. Since $\left|\hat{\theta}_{\mathrm{PEL}}-\theta_{0}\right|_{\infty}=O_{p}\left(\alpha_{n}\right)$ for some $\alpha_{n} \rightarrow 0$ as $n \rightarrow \infty$, if the signal strength of the nonzero components satisfies the condition $\alpha_{n}=o\left(\min _{k \in \mathcal{S}}\left|\theta_{0, k}\right|\right)$, such a support estimation is valid in the sense that $\operatorname{pr}\left\{\operatorname{supp}\left(\hat{\theta}_{\mathrm{PEL}}\right)=\mathcal{S}\right\} \rightarrow 1$ as $n \rightarrow \infty$. 


\subsection{Two inference problems of interest}

We will study two problems thoroughly:

(a) (Inference for multiple components of model parameters) Without loss of generality, we write $\theta=\left(\theta_{\mathcal{M}}^{\mathrm{T}}, \theta_{\mathcal{M}^{c}}^{\mathrm{T}}\right)^{\mathrm{T}}$, where $\theta_{\mathcal{M}} \in \mathbb{R}^{m}$ contains the low-dimensional components of interests, and $\theta_{\mathcal{M}^{c}} \in \mathbb{R}^{p-m}$ contains the nuisance parameters. The construction of confidence regions for $\theta_{\mathcal{M}}$ will be shown in $\S 3 \cdot 1$.

(b) (Over-identification test) When $r>p$, a specification test is proposed in $\S 3.2$ to check the validity of model (1) by testing the hypothesis $H_{0}: E\left\{g_{i}\left(\theta_{0}\right)\right\}=0$ for some $\theta_{0} \in \Theta$ versus $H_{1}: E\left\{g_{i}(\theta)\right\} \neq 0$ for any $\theta \in \Theta$.

In Problem (a) when $m=1$, our method reduces to the special case of constructing a confidence interval for an individual component of $\theta$. More generally when $m>1$, we are estimating the confidence region for multiple components as specified by $\theta_{\mathcal{M}}$. Although $\hat{\theta}_{\mathrm{PEL}}$ and $\hat{\theta}_{\mathrm{PELbc}}$ in (2) and (4) provide consistent estimates for $\theta_{0}$, we cannot use their limiting distributions to solve Problem (a) mainly due to two reasons: (i) $\mathcal{S}$ is generally unknown, and (ii) the limiting distributions of $\hat{\theta}_{\mathrm{PEL}, \mathcal{S}^{c}}$ and $\hat{\theta}_{\mathrm{PELbc}, \mathcal{S}^{c}}$ are also unknown. Problem (b) is known as the over-identification test. The Sargan-Hansen's $J$-test (Sargan, 1958; Hansen, 1982) and the empirical likelihood ratio test (Qin \& Lawless, 1994) can be used for such a purpose when $r$ and $p$ are fixed. When both $r$ and $p$ are less than $n$ or diverge with $n$ at some polynomial rate, by appropriate normalization, the Sargan-Hansen test and the empirical likelihood ratio test may still apply (Chang et al., 2015). However, when $p$ and/or $r$ is greater than $n$, neither applies because they both rely explicitly or implicitly on inverting a large sample covariance matrix that is not of full rank.

\section{Methodology}

\subsection{Inference for low-dimensional components of model parameters}

When $r$ and $p$ are fixed, the profile empirical likelihood approach of Qin \& Lawless (1994) can be applied to solve Problem (a). Specifically, we consider the empirical likelihood function

$$
L(\theta)=\sup \left\{\prod_{i=1}^{n} \pi_{i}: \pi_{i}>0, \sum_{i=1}^{n} \pi_{i}=1, \sum_{i=1}^{n} \pi_{i} g_{i}(\theta)=0\right\}
$$

for any $\theta \in \Theta$, and define the empirical likelihood estimator for $\theta_{0}$ as $\check{\theta}_{n}=\arg \max _{\theta \in \Theta} L(\theta)$. The profile empirical likelihood ratio is defined as $\tilde{\ell}\left(\theta_{\mathcal{M}}\right)=\ell\left(\theta_{\mathcal{M}}, \bar{\theta}_{\mathcal{M}^{c}}\right)-\ell\left(\check{\theta}_{n}\right)$, where $\ell(\theta)=$ $-2 \log \left\{n^{n} L(\theta)\right\}$, and $\bar{\theta}_{\mathcal{M}^{c}}$ minimizes $\ell\left(\theta_{\mathcal{M}}, \theta_{\mathcal{M}^{c}}\right)$ with respect to $\theta_{\mathcal{M}^{c}}$ for a given $\theta_{\mathcal{M}}$. It is well known that $\tilde{\ell}\left(\theta_{0, \mathcal{M}}\right) \rightarrow \chi_{m}^{2}$ in distribution as $n \rightarrow \infty$. Then $\left\{\theta_{\mathcal{M}} \in \mathbb{R}^{m}: \tilde{\ell}\left(\theta_{\mathcal{M}}\right) \leq \chi_{m, 1-\alpha}^{2}\right\}$ provides a $100(1-\alpha) \%$ confidence region for $\theta_{\mathcal{M}}$, where $\chi_{m, 1-\alpha}^{2}$ denotes the $(1-\alpha)$-quantile of chi-square distribution with $m$ degrees of freedom.

The confidence regions constructed by empirical likelihood ratio have several advantages (Hall \& La Scala, 1990). First, empirical likelihood-based confidence regions are data-driven, being free from shape constraints; see the plots of the estimated confidence regions from our simulation in the Supplementary Material. Second, though being nonparametric, empirical likelihood-based confidence regions are Bartlett correctable, so the order of the coverage error can be reduced from $n^{-1}$ to $n^{-2}$ with a simple correction for the mean of empirical likelihood ratio statistics (Chen \& Cui, 2006, 2007). Third, empirical likelihood method requires no further estimations such as the scale and the skewness, which is an appealing convenience for solving high-dimensional 
problems. Fourth, empirical likelihood method can be adapted to construct confidence regions for general smooth functions of the model parameter (Qin \& Lawless, 1995).

Clearly, when both $r$ and $p$ are allowed to diverge with $n$, the profile empirical likelihood approach encounters substantial difficulty. First, calculating $\tilde{\ell}\left(\theta_{\mathcal{M}}\right)$ is challenging due to the fact that it is generally a high-dimensional, non-convex optimization problem. Second, the existing asymptotic analysis on the profile empirical likelihood ratio $\tilde{\ell}\left(\theta_{\mathcal{M}}\right)$ cannot be generalized to highdimensional case. To illustrate, let us first pretend that the truth of the nuisance parameters $\theta_{\mathcal{M}^{c}}$, denoted by $\theta_{0, \mathcal{M}^{c}}$, is known. Then the empirical likelihood for $\theta_{\mathcal{M}} \in \mathbb{R}^{m}$ follows the conventional framework. When $r$ is fixed, the empirical likelihood ratio $\ell\left(\theta_{0}\right)=-2 \log \left\{n^{n} L\left(\theta_{0}\right)\right\} \rightarrow$ $\chi_{r}^{2}$ in distribution as $n \rightarrow \infty$, so $\left\{\theta_{\mathcal{M}} \in \mathbb{R}^{m}: \ell\left(\theta_{\mathcal{M}}, \theta_{0, \mathcal{M}^{c}}\right) \leq \chi_{r, 1-\alpha}^{2}\right\}$ is a valid confidence region for $\theta_{\mathcal{M}}$. If $\theta_{0, \mathcal{M}^{c}}$ is replaced by a $\sqrt{n}$-consistent estimate $\tilde{\theta}_{\mathcal{M}^{c}}$, still keeping $r$ fixed, $\ell\left(\theta_{0, \mathcal{M}}, \tilde{\theta}_{\mathcal{M}^{c}}\right)$ generally converges to some weighted sum of chi-square distributions (Hjort et al., 2009). However, if the estimate $\tilde{\theta}_{\mathcal{M}^{c}}$ converges to $\theta_{0, \mathcal{M}^{c}}$ slower than $n^{-1 / 2}, \ell\left(\theta_{0, \mathcal{M}}, \tilde{\theta}_{\mathcal{M}^{c}}\right)$ generally diverges with probability approaching one (Chang et al., 2013, 2016). When $\theta$ is highdimensional, convergence rate of such estimators is generally slower than $n^{-1 / 2}$. Hence, a naive plug-in of $\tilde{\theta}_{\mathcal{M}^{c}}$ into (5) will not work. A key reason leading to the failure of empirical likelihood with high-dimensional problems is caused by the errors from estimating the nuisance parameters.

Therefore, it is crucial to investigate the impact on empirical likelihood from the estimation of nuisance parameters. In conventional settings with fixed number of model parameters, the first and second order properties of empirical likelihood ratio statistics are documented in Qin \& Lawless (1994), Lazar \& Mykland (1999) and Chen \& Cui (2006, 2007). Hjort et al. (2009) consider nuisance parameters that can be functional-valued and estimated by some nonparametric methods. The work of Bravo et al. (2019) demonstrates that by using estimated influence functions, the chi-squared distributed empirical likelihood ratio statistics can be justified. Nevertheless, it remains little explored in the literature on how to handle high-dimensional nuisance parameters when penalized estimation approaches are used. Though sparse and consistent parameter estimates are achievable with penalized empirical likelihood (Chang et al., 2018), the zero components in the estimates are essentially degenerated and, even worse, their influence functions do not exist, rendering inapplicability of the existing methods. Hence our challenge here is fundamentally different from, for example, functional-valued nuisance parameters where smoothness around the truth leads to uniformly consistent and regular estimations.

To cope with nuisance parameters, we observe that for a consistent estimator $\theta_{\mathcal{M}^{c}}^{*}$ of $\theta_{0, \mathcal{M}^{c}}$

$$
Q_{n}=\bar{g}\left(\theta_{0, \mathcal{M}}, \theta_{\mathcal{M}^{c}}^{*}\right)-\bar{g}\left(\theta_{0}\right)=\left\{\nabla_{\theta_{\mathcal{M}^{c}}} \bar{g}\left(\theta_{0, \mathcal{M}}, \theta_{\mathcal{M}^{c}}^{*}\right)\right\}\left(\theta_{\mathcal{M}^{c}}^{*}-\theta_{0, \mathcal{M}^{c}}\right)+R_{1},
$$

where $R_{1}$ is asymptotically negligible. A strategy here is to find a linear transformation matrix $A_{n}=\left(a_{1}^{n}, \ldots, a_{m}^{n}\right)^{\mathrm{T}} \in \mathbb{R}^{m \times r}$ satisfying $\left|A_{n} Q_{n}\right|_{2}=o_{p}\left(n^{-1 / 2}\right)$, where each $a_{k}^{n}$ is an $r$ dimensional vector. Then by utilizing $f^{A_{n}}(\cdot ; \cdot)=A_{n} g(\cdot ; \cdot)$ as the new $m$-dimensional estimating functions, the empirical likelihood constructed with $f^{A_{n}}(\cdot ; \cdot)$ instead of $g(\cdot ; \cdot)$ can be used for statistical inference for $\theta_{\mathcal{M}}$. Specifically, let $\ell_{A_{n}}^{*}\left(\theta_{\mathcal{M}}\right)=-2 \log \left\{n^{n} L_{A_{n}}^{*}\left(\theta_{\mathcal{M}} ; \theta_{\mathcal{M}^{c}}^{*}\right)\right\}$ with

$$
L_{A_{n}}^{*}\left(\theta_{\mathcal{M}} ; \theta_{\mathcal{M}^{c}}^{*}\right)=\sup \left\{\prod_{i=1}^{n} \pi_{i}: \pi_{i}>0, \sum_{i=1}^{n} \pi_{i}=1, \sum_{i=1}^{n} \pi_{i} f_{i}^{A_{n}}\left(\theta_{\mathcal{M}}, \theta_{\mathcal{M}^{c}}^{*}\right)=0\right\} .
$$

From (6), an ideal choice of $A_{n}$ should be such that $A_{n} \nabla_{\theta_{\mathcal{M}}} \bar{g}\left(\theta_{0, \mathcal{M}}, \theta_{\mathcal{M}^{c}}^{*}\right)$ being small in the sense that each row vector $\left(a_{k}^{n}\right)^{\mathrm{T}}$ of $A_{n}$ satisfies that $\left|\left(a_{k}^{n}\right)^{\mathrm{T}}\left\{\nabla_{\theta_{\mathcal{M}^{c}}} \bar{g}\left(\theta_{0, \mathcal{M}}, \theta_{\mathcal{M}^{c}}^{*}\right)\right\}\right|_{\infty}$ diminishes to 0 as $n \rightarrow \infty$. Or equivalently, rows of $A_{n}$ should be chosen as asymptotically orthogonal to the column space of $\nabla_{\theta_{\mathcal{M}^{c}}} \bar{g}\left(\theta_{0, \mathcal{M}}, \theta_{\mathcal{M}^{c}}^{*}\right)$ - the $r \times(p-m)$ sample gradient matrix with respect to the nuisance parameters. As an additional key consideration, we note that the gradient with 
respect to $\theta_{\mathcal{M}}$ evaluated at $\left(\theta_{0, \mathcal{M}}, \theta_{\mathcal{M}^{c}}^{*}\right)$ should not vanish; otherwise, a flat estimating function at $\theta_{0, \mathcal{M}}$ is not informative. Thus, $A_{n} \nabla_{\theta_{\mathcal{M}}} \bar{g}\left(\theta_{0, \mathcal{M}}, \theta_{\mathcal{M}^{c}}^{*}\right)$ is required to be nonsingular. In practice, the true $\theta_{0, \mathcal{M}}$ is unknown so an estimate, denoted by $\theta_{\mathcal{M}}^{*}$, is needed when constructing $A_{n}$.

By putting the ideas together, we propose to find $A_{n}$ row by row with the optimizations

$$
a_{k}^{n}=\arg \min _{u \in \mathbb{R}^{r}}|u|_{1} \quad \text { s.t }\left|\left\{\nabla_{\theta} \bar{g}\left(\theta^{*}\right)\right\}^{\mathrm{T}} u-\xi_{k}\right|_{\infty} \leq \tau,
$$

where $\theta^{*}=\left\{\left(\theta_{\mathcal{M}}^{*}\right)^{\mathrm{T}},\left(\theta_{\mathcal{M}^{c}}^{*}\right)^{\mathrm{T}}\right\}^{\mathrm{T}}$ is an initial estimate for $\theta_{0}, \tau$ is a tuning parameter, and $\left\{\xi_{k}\right\}_{k=1}^{m}$ are the canonical basis of the linear space $\mathcal{M}_{\xi}=\left\{b=\left(b_{1}, \ldots, b_{p}\right)^{\mathrm{T}}: b_{j}=0\right.$ for any $j=m+$ $1, \ldots, p\}$, i.e., $\xi_{k}$ is chosen such that its $k$-th component is 1 and all other components are 0 . Thus, a $100(1-\alpha) \%$-level confidence region for $\theta_{\mathcal{M}}$ is given as follows:

(i) When $m$ is fixed, $\mathcal{C}_{1-\alpha}=\left\{\theta_{\mathcal{M}} \in \mathbb{R}^{m}: \ell_{A_{n}}^{*}\left(\theta_{\mathcal{M}}\right) \leq \chi_{m, 1-\alpha}^{2}\right\}$ where $\chi_{m, 1-\alpha}^{2}$ is the $(1-\alpha)$ quantile of chi-square distribution with $m$ degrees of freedom.

(ii) When $m$ is diverging, $\mathcal{C}_{1-\alpha}=\left\{\theta_{\mathcal{M}} \in \mathbb{R}^{m}: \ell_{A_{n}}^{*}\left(\theta_{\mathcal{M}}\right) \leq m+z_{1-\alpha}(2 m)^{1 / 2}\right\}$ where $z_{1-\alpha}$ is the $(1-\alpha)$-quantile of standard normal distribution $N(0,1)$. The rationale is that $\left(\chi_{m}^{2}-\right.$ $m) / \sqrt{2 m} \rightarrow N(0,1)$ in distribution as $m \rightarrow \infty$.

To construct $f^{A_{n}}(\cdot ; \cdot)$ in (7), one needs no more than the original estimating functions $g(\cdot ; \cdot)$ and an initial estimate $\theta^{*}$. This strategy is new and effective as it can be generally adapted to handle nuisance parameters with empirical likelihood or as a development of its own interests. Theorem 1 in $\S 4.1$ establishes the validity of the above procedure. Briefly speaking, for a given consistent initial estimate $\theta^{*}$, the estimated confidence region is asymptotically valid as $n \rightarrow \infty$, allowing both $r$ and $p$ to diverge at some exponential rate of $n$. Requiring a consistent initial estimate $\theta^{*}$ is not restrictive and can be broadly satisfied by sparse penalized estimates in cases such as linear models and generalized linear models. For more general problems associated with estimating equations, we advocate to apply $\hat{\theta}_{\text {PEL }}$ given by (2). As an advantage of our method, it does not require the bias-correction step when using the transformed estimating function $f^{A_{n}}(\cdot ; \cdot)$. As comparison, in Zhang \& Zhang (2013) and van de Geer et al. (2014), bias-correction is necessary to construct normal distribution-based confidence intervals in high-dimensional linear models.

Let $\Gamma=E\left\{\nabla_{\theta} g_{i}\left(\theta_{0}\right)\right\}$. We note that the existence of $a_{k}$ such that $\Gamma^{\mathrm{T}} a_{k}=\xi_{k}$ is an elementary requirement. Since $\Gamma \in \mathbb{R}^{r \times p}$ with $r \geq p, a_{k}$ may not be unique. A major challenge of the theoretical analysis is the identifiability of $A_{n}$ from (8) for high-dimensional problems. We impose the following regularity condition:

Condition 1. For each $k=1, \ldots, m$, there is a nonrandom $a_{k}$ satisfying $\Gamma^{\mathrm{T}} a_{k}=\xi_{k},\left|a_{k}\right|_{1} \leq$ $C_{1}$ for some uniform constant $C_{1}>0$, and $\max _{1 \leq k \leq m}\left|a_{k}^{n}-a_{k}\right|_{1}=O_{p}\left(\omega_{n}\right)$ for some $\omega_{n} \rightarrow 0$.

Let $\hat{\Gamma}_{n}=\nabla_{\theta} \bar{g}\left(\theta^{*}\right)$. It follows from the existence of $a_{k}$ that $\xi_{k}=\hat{\Gamma}_{n}^{\mathrm{T}} a_{k}+\left(\Gamma-\hat{\Gamma}_{n}\right)^{\mathrm{T}} a_{k}=$ $\hat{\Gamma}_{n}^{\mathrm{T}} a_{k}+e_{k}$. Under some mild conditions, $\left|\hat{\Gamma}_{n}-\Gamma\right|_{\infty} \rightarrow 0$ in probability. This, together with the assumption that $\left|a_{k}\right|_{1} \leq C_{1}$, implies that $e_{k}$ is stochastically small uniformly over all its components such that $\left|e_{k}\right|_{\infty}=o_{p}(1)$. This can be seen as an attempt to recover a nonrandom $a_{k}$ with no noise asymptotically (Candès \& Tao, 2007; Bickel et al., 2009). Thus, $a_{k}^{n}$ from (8) satisfies $\left|a_{k}^{n}-a_{k}\right|_{1} \rightarrow 0$ in probability if $\hat{\Gamma}_{n}$ satisfies the routine conditions for sparse signal recovering. It can be shown that $C_{1}$ in Condition 1 can be replaced by some diverging sequence $\gamma_{n}$ and our main results remain valid. Theorem 1 in $\S 4.1$ indicates that it only requires $\omega_{n}$ to satisfy $m \omega_{n}^{2}(m+\log r)=o(1)$ for the validity of our procedure. For the just-identified case where $r=p$, our assumption on the existence of $\Gamma^{\mathrm{T}} a_{k}=\xi_{k}$ is weaker than that of Neykov et al. (2018), which assumes $\Gamma$ to be invertible to make $a_{k}=\Gamma^{-1} \xi_{k}$ unique. 
Our statistical inferential procedure can be extended to broader cases of interest. For a general function $S\left(\theta_{\mathcal{M}}\right) \in \mathbb{R}^{q}$ of a specified $\theta_{\mathcal{M}}$, the formulation of Qin \& Lawless (1995) can be applied to construct the confidence region for $S\left(\theta_{\mathcal{M}}\right)$ :

$$
\mathcal{C}_{1-\alpha}=\left\{v \in \mathbb{R}^{q}: \min _{\theta_{\mathcal{M}}: S\left(\theta_{\mathcal{M}}\right)=v} \ell_{A_{n}}^{*}\left(\theta_{\mathcal{M}}\right) \leq \chi_{q, 1-\alpha}^{2}\right\}
$$

For univariate and monotone transformation $S(\cdot)$, the confidence region with empirical likelihood has the invariant property (Hall \& La Scala, 1990). In the special case $S\left(\theta_{\mathcal{M}}\right)=L \theta_{\mathcal{M}}$ with $L \in \mathbb{R}^{q \times m}$, i.e., $q$ linear combinations of $\theta_{\mathcal{M}}$, the validity of such defined confidence region can be established following the same idea of our analysis.

We note that empirical likelihood with over-identified general estimating equations may provide a unique opportunity for enhancing the accuracy of statistical inference. For the inference of $m$-dimensional components $\theta_{\mathcal{M}}$, one may opt to find $\tilde{m}(\tilde{m} \geq m)$ linear combinations of the original estimating function. The rationale is that $\Gamma^{\mathrm{T}} u=\xi_{k}$, as in Condition 1, may yield multiple linearly independent sparse solutions. Practically, an option is to implement (8) sequentially: upon finding a solution $a_{k}^{n, 1}$, one runs (8) to find another solution $a_{k}^{n, 2}$ subject to an additional linear constrain such that $\left(a_{k}^{n, 1}\right)^{\mathrm{T}} a_{k}^{n, 2}=0$. Using over-identification $(\tilde{m}>m)$ for $\theta_{\mathcal{M}}$ is beneficial for improving the accuracy of statistical inference (Qin \& Lawless, 1994). As shown in our simulation in $\S 5$, such improvement is substantial.

\subsection{Over-identification test}

Over-identification provides an opportunity to develop a statistical test for checking the validity of model specification. In low-dimensional cases, the Sargan-Hansen's $J$-test (Sargan, 1958; Hansen, 1982) and the empirical likelihood ratio test (Qin \& Lawless, 1994) can be used. Qin \& Lawless (1994) shows that $\ell\left(\check{\theta}_{n}\right)=-2 \log \left\{n^{n} L\left(\check{\theta}_{n}\right)\right\} \rightarrow \chi_{r-p}^{2}$ in distribution under $H_{0}$, where $\check{\theta}_{n}$ is the maximizer of $L(\theta)$ in (5). It can be shown that the Sargan-Hansen's $J$ statistic is firstorder equivalent to the empirical likelihood ratio statistic $\ell\left(\check{\theta}_{n}\right)$, therefore they share the same limiting distribution. When the paradigm shifts to high-dimensional settings, the asymptotic quadratic form no longer holds and the limiting $\chi_{r-p}^{2}$ distribution becomes invalid.

Our over-identification test is developed from the marginal empirical likelihood ratios. Given $\hat{\theta}_{n}$, a consistent estimate of $\theta_{0}$ under $H_{0}$ (will be specified later), we define the marginal empirical likelihood ratio for the $j$-th estimating function $g_{j}(\cdot ; \cdot)$ in $g(\cdot ; \cdot)$ as

$$
\ell_{j}\left(\hat{\theta}_{n}\right)=2 \max _{\lambda \in \hat{\Lambda}_{n, j}} \sum_{i=1}^{n} \log \left\{1+\lambda g_{i, j}\left(\hat{\theta}_{n}\right)\right\},
$$

where $\hat{\Lambda}_{n, j}=\left\{\lambda \in \mathbb{R}: \lambda g_{i, j}\left(\hat{\theta}_{n}\right) \in \mathcal{U}\right.$ for any $\left.i=1, \ldots, n\right\}$ with an open interval $\mathcal{U}$ containing zero. Based on $\left\{\ell_{j}\left(\hat{\theta}_{n}\right)\right\}_{j=1}^{r}$, we propose the following test statistic

$$
T_{n}=\max _{j \in \mathcal{J}} \ell_{j}\left(\hat{\theta}_{n}\right)
$$

where $\mathcal{J}$ is a prescribed index set with $|\mathcal{J}|=q$. Since the calculation of $\ell_{j}\left(\hat{\theta}_{n}\right)$ only involves univariate optimizations, calculating $T_{n}$ is highly scalable and can be done efficiently. The intuition of (9) is that when $H_{0}$ is true, each $\ell_{j}\left(\hat{\theta}_{n}\right)$ should take a relatively small value. In contrast, when $H_{0}$ is violated, one expects that at least some $\ell_{j}\left(\hat{\theta}_{n}\right)$ 's to be large.

The selection of index set $\mathcal{J}$ in (9) is the key to developing a powerful procedure for highdimensional over-identification test. In low-dimensional cases, a natural choice of $\mathcal{J}$ is to include all $r$ estimating functions. However, additional consideration is necessary when dealing 
with high-dimensional problems. To illustrate the idea, we add the subscript $\mathcal{J}$ in $T_{n}$ here to emphasize the dependency. In our method, the $\alpha$-level critical value for $T_{n, \mathcal{J}}$ is selected as the $(1-\alpha)$-quantile of the distribution of $\left|\hat{G}_{\mathcal{J}}\right|_{\infty}^{2}$, where $\hat{G}_{\mathcal{J}}$ follows some $q$-dimensional multivariate normal distribution. Let $j_{\sharp}=\arg \max _{1 \leq j \leq r} \ell_{j}\left(\hat{\theta}_{n}\right)$. For any two different index sets $\mathcal{J}_{1}$ and $\mathcal{J}_{2}$ satisfying $j_{\sharp} \in \mathcal{J}_{1} \cap \mathcal{J}_{2}$ and $\mathcal{J}_{1} \subset \mathcal{J}_{2}$, it is easy to see that $T_{n, \mathcal{J}_{1}}=T_{n, \mathcal{J}_{2}}$. Due to the fact that $\hat{G}_{\mathcal{J}_{1}}$ is a subvector of $\hat{G}_{\mathcal{J}_{2}}$, the $(1-\alpha)$-quantile of the distribution of $\left|\hat{G}_{\mathcal{J}_{1}}\right|_{\infty}^{2}$ will be no larger than that of $\left|\hat{G}_{\mathcal{J}_{2}}\right|_{\infty}^{2}$. Hence, when too many components are included in constructing the test statistic, the associated critical value inevitably becomes too large, which will lead to power loss. To obtain a powerful test, we only need to select a small index set $\mathcal{J}$ with $j_{\sharp}$ being included to best maintain the signal for detecting the violation of $H_{0}$; see also $\S 2.3$ of Chang et al. (2017) for more discussion on such a phenomenon of $L_{\infty}$-type test statistic. Further, results in Chang et al. $(2013,2016)$ show that $\ell_{j}\left(\hat{\theta}_{n}\right)$ diverges fast if $\left|\bar{g}_{j}\left(\hat{\theta}_{n}\right)\right|$ does not converge to zero fast enough - the signal from violating $H_{0}$ that the over-identification test intends to detect. Thus, one should ideally include in the index set $\mathcal{J}$ those $j$ 's with large $\left|\bar{g}_{j}\left(\hat{\theta}_{n}\right)\right|$. The selection of $\mathcal{J}$ will be elaborated more at the end of this section.

Obviously, the test statistic $T_{n}$ in (9) depends on the estimate $\hat{\theta}_{n}$. Recall $\mathcal{S}=\operatorname{supp}\left(\theta_{0}\right)=\{1 \leq$ $\left.k \leq p: \theta_{0, k} \neq 0\right\}$ with $|\mathcal{S}|=s$. Our theoretical analysis requires $\hat{\theta}_{n}$ to satisfy the following two properties under $H_{0}$ :

(i) $\hat{\theta}_{n, \mathcal{S}}-\theta_{0, \mathcal{S}}=n^{-1} \sum_{i=1}^{n} m\left(X_{i} ; \theta_{0}\right)+\Delta_{n}$ with $\left|\Delta_{n}\right|_{2}=o_{p}\left(n^{-1 / 2}\right)$,

(ii) $\operatorname{pr}\left(\hat{\theta}_{n, \mathcal{S}^{c}}=0\right) \rightarrow 1$ as $n \rightarrow \infty$,

where $m(\cdot ; \cdot)$ is the $s$-dimensional influence function of $\hat{\theta}_{n, \mathcal{S}}$. To require $\left|\Delta_{n}\right|_{2}=o_{p}\left(n^{-1 / 2}\right)$ in Property (i) is not stringent and can be satisfied by penalized likelihood estimates up to a bias correction (Fan \& Li, 2001). Property (ii) is the oracle property. As seen below, Property (ii) is not essential but more involved characterization is required without it. In special cases including the linear and generalized linear models, we recommend applying bias correction or re-fitting the selected model to obtain less biased estimates, for example, using the method in Belloni \& Chernozhukov (2013). For more general models with estimating equations, $\hat{\theta}_{n}$ can be chosen as the bias-corrected estimate $\hat{\theta}_{\text {PELbc }}$ given by (4) in $\S 2 \cdot 1$, which meets the requirements by Proposition 2 in $\S 2 \cdot 1$.

Denote $\hat{V}_{\mathcal{J}}\left(\hat{\theta}_{n}\right)=n^{-1} \sum_{i=1}^{n} g_{i, \mathcal{J}}\left(\hat{\theta}_{n}\right)^{\otimes 2}$ and $V_{\mathcal{J}}\left(\theta_{0}\right)=E_{\mathcal{J}}\left\{g_{i, \mathcal{J}}\left(\theta_{0}\right)^{\otimes 2}\right\}$. Here when we define $V_{\mathcal{J}}\left(\theta_{0}\right)$ we only take the expectation with respect to $X_{i}$ and treat the index set $\mathcal{J}$ as given, which itself might be random. For any $j \in \mathcal{J}$, let $\hat{\sigma}_{j}^{2}\left(\hat{\theta}_{n}\right)=n^{-1} \sum_{i=1}^{n} g_{i, j}^{2}\left(\hat{\theta}_{n}\right)$. Based on the well-known self-Studentized property of the empirical likelihood ratio, it can be shown under $H_{0}$ that $\sup _{j \in \mathcal{J}}\left|\ell_{j}\left(\hat{\theta}_{n}\right)-n\left\{\bar{g}_{j}\left(\hat{\theta}_{n}\right)\right\}^{2} \hat{\sigma}_{j}^{-2}\left(\hat{\theta}_{n}\right)\right|=o_{p}(1)$. By expanding $\bar{g}_{\mathcal{J}}\left(\hat{\theta}_{n}\right)$ around $\theta_{0}$, it holds that $n^{1 / 2}\left[\operatorname{diag}\left\{\hat{V}_{\mathcal{J}}\left(\hat{\theta}_{n}\right)\right\}\right]^{-1 / 2} \bar{g}_{\mathcal{J}}\left(\hat{\theta}_{n}\right)=n^{-1 / 2} \sum_{i=1}^{n} w_{i}\left(\theta_{0}\right)+\tilde{\Delta}_{n}$, where $w_{i}\left(\theta_{0}\right)=$ $\left[\operatorname{diag}\left\{V_{\mathcal{J}}\left(\theta_{0}\right)\right\}\right]^{-1 / 2}\left\{g_{i, \mathcal{J}}\left(\theta_{0}\right)+\left[E_{\mathcal{J}}\left\{\nabla_{\theta_{\mathcal{S}}} g_{i, \mathcal{J}}\left(\theta_{0}\right)\right\}\right] m_{i}\left(\theta_{0}\right)\right\}$ and $\left|\tilde{\Delta}_{n}\right|_{\infty}=o_{p}\left(n^{-1 / 2}\right)$. Following the idea of Gaussian approximation (Chernozhukov et al., 2017), we can approximate the distribution of $T_{n}=\max _{j \in \mathcal{J}} \ell_{j}\left(\hat{\theta}_{n}\right)$ by that of $|\hat{G}|_{\infty}^{2}$, where $\hat{G} \sim N(0, \hat{W})$ for some $\hat{W}$.

Since $\hat{\theta}_{n}$ is estimated from the data $\mathcal{X}_{n}=\left\{X_{1}, \ldots, X_{n}\right\}$, its influence function $m_{i}(\cdot)$ and the estimating function $g_{i}(\cdot)$ are dependent. As we have discussed below Proposition 2 in $\S 2 \cdot 1$, the unknown index set $\mathcal{S}$ can be consistently estimated. To simplify the notation and without lose of generality, we assume $\mathcal{S}$ is known. Otherwise, we can replace it in practice by $\hat{\mathcal{S}}=\operatorname{supp}\left(\hat{\theta}_{\mathrm{PEL}}\right)$ for $\hat{\theta}_{\mathrm{PEL}}$ in (2). To elaborate with details on $\hat{W}$, we present the framework by selecting $\hat{\theta}_{n}$ as

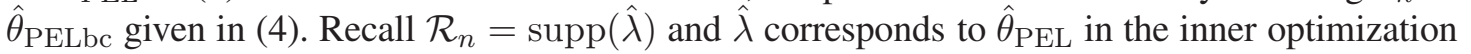
of (2). Singling out $\mathcal{R}_{n}$ here is necessary to concretely present a synthetic framework. 
To avoid loss of generality, we do not impose any relationship between the two index sets $\mathcal{J}$ and $\mathcal{R}_{n}$ in our theoretical analysis. Let $\mathcal{I}=\mathcal{R}_{n} \cup \mathcal{J}$ and we note that both the estimating functions in $g(\cdot ; \cdot)$ indexed by $\mathcal{I}$ and the covariance matrix of $\hat{\theta}_{n, \mathcal{S}}$ contribute to the joint distribution of $\left\{\ell_{j}\left(\hat{\theta}_{n}\right)\right\}_{j \in \mathcal{J}}$; see Lemmas 5 and 6 in the Supplementary Material. For any $\mathcal{L} \subset\{1, \ldots, r\}$, we define $V_{\mathcal{L}}\left(\theta_{0}\right)=E_{\mathcal{L}}\left\{g_{i, \mathcal{L}}\left(\theta_{0}\right)^{\otimes 2}\right\}$ and $J_{\mathcal{L}}=\left\{\left[E_{\mathcal{L}}\left\{\nabla_{\theta_{\mathcal{S}}} g_{i, \mathcal{L}}\left(\theta_{0}\right)\right\}\right]^{\mathrm{T}} V_{\mathcal{L}}^{-1 / 2}\left(\theta_{0}\right)\right\}^{\otimes 2}$. Again, both expectations are taken with respect to $X_{i}$ and the index set $\mathcal{L}$ are treated as given. To ensure the validity of $\hat{W}$ given in (12), we re-write $g(\cdot ; \cdot)$ as

$$
g(\cdot ; \cdot)=\left\{g_{\mathcal{R}_{n} \cap \mathcal{J}}(\cdot ; \cdot)^{\mathrm{T}}, g_{\mathcal{R}_{n} \cap \mathcal{J}^{c}}(\cdot ; \cdot)^{\mathrm{T}}, g_{\mathcal{R}_{n}^{c} \cap \mathcal{J}}(\cdot, \cdot)^{\mathrm{T}}, g_{\mathcal{I}^{c}}(\cdot ; \cdot)^{\mathrm{T}}\right\}^{\mathrm{T}} .
$$

Define $B=\left[E_{\mathcal{J}}\left\{\nabla_{\theta_{\mathcal{S}}} g_{i, \mathcal{J}}\left(\theta_{0}\right)\right\}\right] J_{\mathcal{R}_{n}}^{-1}\left[E_{\mathcal{R}_{n}}\left\{\nabla_{\theta_{\mathcal{S}}} g_{i, \mathcal{R}_{n}}\left(\theta_{0}\right)\right\}\right]^{\mathrm{T}} V_{\mathcal{R}_{n}}^{-1}\left(\theta_{0}\right)$ with blocks:

$$
B=\left(\begin{array}{ll}
B_{11} & B_{12} \\
B_{21} & B_{22}
\end{array}\right)
$$

where $B_{11}$ and $B_{22}$ are $\left|\mathcal{R}_{n} \cap \mathcal{J}\right| \times\left|\mathcal{R}_{n} \cap \mathcal{J}\right|$ and $\left|\mathcal{R}_{n}^{c} \cap \mathcal{J}\right| \times\left|\mathcal{R}_{n} \cap \mathcal{J}^{c}\right|$ matrices. Let

$$
\hat{Q}=\left(\begin{array}{ccc}
I_{\left|\mathcal{R}_{n} \cap \mathcal{J}\right|}-\hat{B}_{11} & -\hat{B}_{12} & 0 \\
-\hat{B}_{21} & -\hat{B}_{22} & I_{\left|\mathcal{R}_{n}^{c} \cap \mathcal{J}\right|}
\end{array}\right)
$$

where $I_{k}$ is the identity matrix with order $k$, and $\hat{B}_{i j}(i, j=1,2)$ are the corresponding estimates of $B_{i j}$ in the matrix $\hat{B}=\left\{\nabla_{\theta_{\mathcal{S}}} \bar{g}_{\mathcal{J}}\left(\hat{\theta}_{n}\right)\right\} \hat{J}_{*, \mathcal{R}_{n}}^{-1}\left\{\nabla_{\theta_{\mathcal{S}}} \bar{g}_{\mathcal{R}_{n}}\left(\hat{\theta}_{n}\right)\right\}^{\mathrm{T}} \hat{V}_{\mathcal{R}_{n}}^{-1}\left(\hat{\theta}_{n}\right)$ with $\hat{J}_{*, \mathcal{R}_{n}}=$ $\left[\left\{\nabla_{\theta_{\mathcal{S}}} \bar{g}_{\mathcal{R}_{n}}\left(\hat{\theta}_{n}\right)\right\}^{\mathrm{T}} \hat{V}_{\mathcal{R}_{n}}^{-1 / 2}\left(\hat{\theta}_{n}\right)\right]^{\otimes 2}$. Last, we define

$$
\hat{W}=\left\{\left[\operatorname{diag}\left\{\hat{V}_{\mathcal{J}}\left(\hat{\theta}_{n}\right)\right\}\right]^{-1 / 2} \hat{Q} \hat{V}_{\mathcal{I}}^{1 / 2}\left(\hat{\theta}_{n}\right)\right\}^{\otimes 2}
$$

with $\hat{V}_{\mathcal{J}}\left(\hat{\theta}_{n}\right)=n^{-1} \sum_{i=1}^{n} g_{i, \mathcal{J}}\left(\hat{\theta}_{n}\right)^{\otimes 2}$ and $\hat{V}_{\mathcal{I}}\left(\hat{\theta}_{n}\right)=n^{-1} \sum_{i=1}^{n} g_{i, \mathcal{I}}\left(\hat{\theta}_{n}\right)^{\otimes 2}$.

For a given $\alpha \in(0,1)$, the critical value is given by

$$
\hat{\mathrm{cv}}_{\alpha}=\inf \left\{t \in \mathbb{R}: \operatorname{pr}\left(|\hat{G}|_{\infty}^{2}>t \mid \mathcal{X}_{n}\right) \leq \alpha\right\}
$$

where $\hat{G} \sim N(0, \hat{W})$ with $\hat{W}$ defined in (12). We reject $H_{0}$ if $T_{n}>\hat{c v}_{\alpha}$. Furthermore, we note that $c \hat{v}_{\alpha}$ can be conveniently obtained by simulation with $\hat{W}$ obtained from data. That is, one can generate independent $\hat{G}_{1}, \ldots, \hat{G}_{M}$ from $N(0, \hat{W})$ for a large $M$ and approximate $\hat{c v}_{\alpha}$ in (13) by $\hat{c v}_{\alpha, M}=\inf \left\{x \in \mathbb{R}: \hat{F}_{M}(x) \geq 1-\alpha\right\}$ where $\hat{F}_{M}(x)=M^{-1} \sum_{b=1}^{M} I\left(\left|\hat{G}_{b}\right|_{\infty}^{2} \leq x\right)$. The validity of the test is established in $\S 4 \cdot 2$. Theorem 2 justifies that the size of the test is asymptotically $\alpha$ under $H_{0}$, and Theorem 3 elucidates the power of the test when $H_{0}$ is violated.

This section is concluded with a final remark that $\mathcal{R}_{n}$ from (2) is an ideal candidate for $\mathcal{J}$ if $\hat{\theta}_{n}$ is selected to be $\hat{\theta}_{\text {PELbc }}$. As we have discussed before, the index set $\mathcal{J}$ should include those $j$ 's with large $\left|\bar{g}_{j}\left(\hat{\theta}_{n}\right)\right|$. Proposition 3 in Chang et al. (2018) shows that components $g_{j}(\cdot ; \cdot)$ 's with large value in $\left|\bar{g}_{j}\left(\hat{\theta}_{n}\right)\right|$ are included in $\mathcal{R}_{n}$. Furthermore, under $H_{1}$, if $E\left\{g_{i, j}\left(\hat{\theta}_{n}\right)\right\} \neq 0$ for some $j$, its sample counterpart $\left|\bar{g}_{j}\left(\hat{\theta}_{n}\right)\right|$ tends to take some large value, and hence the corresponding index would fall into $\mathcal{R}_{n}$. In practice, we recommend using $\mathcal{R}_{n}$ for the over-identification test, which is the one implemented in our numerical studies. Our simulation in $\S 5.2$ shows that the over-identification test performs very well. By choosing $\mathcal{J}$ in (9) as $\mathcal{R}_{n}$, the test is powerful compared with the one using all the estimating functions, especially when $r$ is large. 


\section{THEORETICAL ANALYSIS}

\subsection{Inference for low-dimensional components}

To establish theoretical guarantees for the validity of the confidence sets $\mathcal{C}_{1-\alpha}$ given in $\S 3 \cdot 1$, we assume the following regularity conditions.

Condition 2. For any $X$ and $j=1, \ldots, p, g_{j}(X ; \theta)$ is twice continuously differentiable with respect to $\theta$. It holds that $\sup _{\theta \in \Theta} \max _{1 \leq j \leq r} \max _{1 \leq l \leq p} n^{-1} \sum_{i=1}^{n}\left|\partial g_{i, j}(\theta) / \partial \theta_{l}\right|^{2}=O_{p}(1)$ and $\sup _{\theta \in \Theta} \max _{1 \leq j \leq r} \max _{1 \leq l_{1}, l_{2} \leq p} n^{-1} \sum_{i=1}^{n}\left|\partial^{2} g_{i, j}(\theta) / \partial \theta_{l_{1}} \partial \theta_{l_{2}}\right|=O_{p}(1)$.

Condition 3. It holds that $\max _{1 \leq j \leq r} E\left\{\sup _{\theta \in \Theta}\left|g_{i, j}(\theta)\right|^{\gamma}\right\}<C_{2}$ for some uniform constants $C_{2}>0$ and $\gamma>4$.

Condition 4. It holds that $\max _{1 \leq j \leq r} n^{-1} \sum_{i=1}^{n}\left|g_{i, j}\left(\theta_{0}\right)\right|^{2}=O_{p}(1)$.

Condition 5. Eigenvalues of $E\left\{g_{i}\left(\theta_{0}\right)^{\otimes 2}\right\}$ are uniformly bounded away from zero and infinity.

Condition 2 is standard on the first and second order derivations of $g(\cdot ; \cdot)$, ensuring its smoothness. If there exist two uniform envelope functions $B_{n, 1}(\cdot)$ and $B_{n, 2}(\cdot)$ with $E\left\{B_{n, 1}^{2}\left(X_{i}\right)\right\}<\infty$ and $E\left\{B_{n, 2}\left(X_{i}\right)\right\}<\infty$ such that $\left|\partial g_{j}(X ; \theta) / \partial \theta_{l}\right| \leq B_{n, 1}(X)$ and $\left|\partial^{2} g_{j}(X ; \theta) / \partial \theta_{l_{1}} \partial \theta_{l_{2}}\right| \leq$ $B_{n, 2}(X)\left(j=1, \ldots, r ; l, l_{1}, l_{2}=1, \ldots, p\right)$ for any $\theta \in \Theta$, then Condition 2 holds automatically. More generally, if there exist envelop functions $B_{n, j l}(\cdot)$ such that $\left|\partial g_{j}(X ; \theta) / \partial \theta_{l}\right|^{2} \leq B_{n, j l}(X)$ $(j=1, \ldots, r ; l=1, \ldots, p)$ for any $\theta \in \Theta$, and $\left|E\left\{B_{n, j l}^{k}\left(X_{i}\right)\right\}\right| \leq H_{1} k ! H_{2}^{k-2}$ for any $k \geq 2$, where $H_{1}$ and $H_{2}$ are two uniform positive constants independent of $j$ and $l$, then Theorem 2.8 in Petrov (1995) implies that $\sup _{1 \leq j \leq r} \sup _{1 \leq l \leq p} n^{-1} \sum_{i=1}^{n} B_{n, j l}\left(X_{i}\right)=O_{p}(1)$, provided $\log (r p)=o(n)$, then Condition 2 holds as well. Conditions 3 and 4 put constraints on the moments of estimating functions. In fact, the order $O_{p}(1)$ required in Conditions 2 and 4 can be replaced by $O_{p}\left(\varpi_{n}\right)$ with some diverging sequence $\varpi_{n}$, and our main results remain valid. We use $O_{p}(1)$ here for the ease of presentation. Condition 5 ensures the non-singularity of the covariance matrix of $g_{i}\left(\theta_{0}\right)$. Under those conditions, we then have the following theorem.

THEOREM 1. Under Conditions 1-5, if $\left|\theta_{\mathcal{M}}^{*}-\theta_{0, \mathcal{M}}\right|_{1}=O_{p}\left(\xi_{1, n}\right)$ and $\left|\theta_{\mathcal{M}^{c}}^{*}-\theta_{0, \mathcal{M}^{c}}\right|_{1}=$ $O_{p}\left(\xi_{2, n}\right)$ for some $\xi_{1, n} \rightarrow 0$ and $\xi_{2, n} \rightarrow 0$, the following results hold:

(i) if $m$ is fixed, then $\ell_{A_{n}}^{*}\left(\theta_{0, \mathcal{M}}\right) \rightarrow \chi_{m}^{2}$ in distribution as $n \rightarrow \infty$, provided that $n \xi_{2, n}^{2}\left(\tau^{2}+\right.$ $\left.\xi_{1, n}^{2}+\xi_{2, n}^{2}\right)=o(1)$ and $\omega_{n}^{2} \log r=o(1)$;

(ii) if $m$ diverges with $n$, then $(2 m)^{-1 / 2}\left\{\ell_{A_{n}}^{*}\left(\theta_{0, \mathcal{M}}\right)-m\right\} \rightarrow N(0,1)$ in distribution as $n \rightarrow \infty$, provided that $m \xi_{2, n}=o(1), m \omega_{n}^{2}(m+\log r)=o(1), m^{3} n^{2 / \gamma-1}=o(1)$, and $m n \xi_{2, n}^{2}\left(\tau^{2}+\right.$ $\left.\xi_{1, n}^{2}+\xi_{2, n}^{2}\right)=o(1)$.

To ensure the validity of the inferential procedure in $\S 3 \cdot 1$, a consistent initial estimate $\theta^{*}$ is required in Theorem 1 . Theorem 1 also suggests that a faster convergence rate of $\theta^{*}$ would allow higher dimensionality of $r$. Define $s^{*}=\left|\left\{1 \leq k \leq m: \theta_{0, k} \neq 0\right\}\right|$ and select $\theta^{*}$ as $\hat{\theta}_{\text {PEL }}$ given by (2). It follows immediately from Proposition 1 that $\xi_{1, n}=s^{*} \alpha_{n}$ and $\xi_{2, n}=\left(s-s^{*}\right) \alpha_{n}$. Theorem 1 holds provided that $m\left(s-s^{*}\right) \alpha_{n}=o(1), m \omega_{n}^{2}(m+\log r)=o(1), m^{3} n^{2 / \gamma-1}=o(1)$, $m n \tau^{2}\left(s-s^{*}\right)^{2} \alpha_{n}^{2}=o(1)$, and $m n s^{2}\left(s-s^{*}\right)^{2} \alpha_{n}^{4}=o(1)$. For the bias-corrected penalized empirical likelihood estimator $\hat{\theta}_{\mathrm{PELbc}}$ in (4) of Chang et al. (2018), $\sqrt{n}$-consistency is achievable for estimating each component of $\theta_{0, \mathcal{S}}$. In such a case, $\xi_{1, n}=s^{*} n^{-1 / 2}$ and $\xi_{2, n}=\left(s-s^{*}\right) n^{-1 / 2}$, and Theorem 1 holds when $m \omega_{n}^{2}(m+\log r)=o(1), m^{3} n^{2 / \gamma-1}=o(1), m \tau^{2}\left(s-s^{*}\right)^{2}=o(1)$, and $m\left(s^{2}+m\right)\left(s-s^{*}\right)^{2} n^{-1}=o(1)$. In addition, if $m$ is fixed, Theorem 1 holds if $\omega_{n}^{2} \log r=$ 
$o(1), s^{2} \tau^{2}=o(1)$, and $s^{4} n^{-1}=o(1)$. Therefore, with a polynomial decay rate $\omega_{n}$ when approximating $a_{k}$ in Condition 1, our method accommodates exponentially diverging $r$ as $n \rightarrow \infty$.

Here are some exemplary scenarios regarding the above conditions. In a just-identified case, let us consider linear regression $Y_{i}=W_{i}^{\mathrm{T}} \theta_{0}+\varepsilon_{i}$, where $\varepsilon_{i}$ is independent of predictor variables $W_{i}=\left(W_{i, 1}, \ldots, W_{i, p}\right)^{\mathrm{T}} \in \mathbb{R}^{p}$, and the estimating function is $g\left(X_{i} ; \theta\right)=W_{i}\left(Y_{i}-W_{i}^{\mathrm{T}} \theta\right)$ with $X_{i}=\left(Y_{i}, W_{i}^{\mathrm{T}}\right)^{\mathrm{T}}$. Condition 2 is equivalent to $\max _{1 \leq j_{1}, j_{2} \leq p} n^{-1} \sum_{i=1}^{n} W_{i, j_{1}}^{2} W_{i, j_{2}}^{2}=O_{p}(1)$. Then, Conditions 2-4 hold if $W_{i}$ and $\varepsilon_{i}$ are sub-Gaussian and $\log p=o(n)$. In an example of over-identified case, we consider a linear regression model $Y_{i}=W_{i}^{\mathrm{T}} \theta_{0}+\varepsilon_{i}$ with instrumental variables $Z_{i} \in \mathbb{R}^{r}$ for $r>p$ and the estimating function is $g\left(X_{i} ; \theta\right)=Z_{i}\left(Y_{i}-W_{i}^{\mathrm{T}} \theta\right)$. Analogously to the just-identified case, Condition 2-5 are met if $W_{i}, Z_{i}$, and $\varepsilon_{i}$ are sub-Gaussian, $\log p=o(n)$, and the eigenvalues of $E\left(\varepsilon_{i}^{2} Z_{i}^{\otimes 2}\right)$ are uniformly bounded away from zero and infinity. If $\varepsilon_{i}$ is independent of $Z_{i}$, the last requirement is equivalent to the boundedness of the eigenvalues of $\operatorname{cov}\left(Z_{i}\right)$.

\subsection{Over-identification test}

Let $q=|\mathcal{J}|$ and $h_{n}=\left|\mathcal{R}_{n}\right|$. To investigate the properties of the over-identification test in $\S$ $3 \cdot 2$, we impose the following condition.

Condition 6. With $\gamma$ specified in Condition 3, there is a uniform constant $C_{3}>0$ such that $E\left\{\left|\partial g_{i, j}\left(\theta_{0}\right) / \partial \theta_{l}\right|^{\gamma}\right\}<C_{3}$ for any $j=1, \ldots, r$ and $l=1, \ldots, p$. In addition, assume

$$
\sup _{\theta \in \Theta} \max _{j \in \mathcal{J}} \frac{1}{n} \sum_{i=1}^{n}\left|g_{i, j}(\theta)\right|^{\gamma}=O_{p}(1) \text {. }
$$

The following theorem is for the type I error of the proposed over-identification test.

THEOREM 2. Assume $B$ defined in (10) to satisfy that $\|B\|_{\infty}$ is bounded away from infinity, and all the eigenvalues of $\left(\left[E_{\mathcal{R}_{n}}\left\{\nabla_{\theta_{\mathcal{S}}} g_{i, \mathcal{R}_{n}}\left(\theta_{0}\right)\right\}\right]^{\mathrm{T}}\right)^{\otimes 2}$ and $\left(\left[E_{\mathcal{J}}\left\{\nabla_{\theta_{\mathcal{S}}} g_{i, \mathcal{J}}\left(\theta_{0}\right)\right\}\right]^{\mathrm{T}}\right)^{\otimes 2}$ are uniformly bounded away from zero and infinity. Select $\hat{\theta}_{n}=\hat{\theta}_{\mathrm{PELbc}}$ defined as (4) with Proposition 2 in $\S 2 \cdot 1$ being satisfied. Let $h_{n} \geq s$ and Conditions 2,5 and 6 hold. If $s^{3} h_{n}^{2} \log ^{4} q=$ $o(n), s h_{n}^{2}\left(\log h_{n}\right) \log ^{4} q=o(n), s^{6} \log ^{2} q=o(n), s^{2} h_{n} \log ^{5} q=o(n), n \phi_{n}^{2} \log q=o(1)$, and $q^{2}(\log n)^{3 \gamma+3}=o\left(n^{\gamma-2}\right)$, then $\sup _{0<\alpha<1}\left|\operatorname{pr}_{H_{0}}\left(T_{n}>\hat{c v}_{\alpha}\right)-\alpha\right| \rightarrow 0$ as $n \rightarrow \infty$, where $\hat{c}_{\alpha}$ is estimated in (13).

Theorem 2 shows the type I error of the proposed over-identification test is approximately $\alpha$. If we select $\mathcal{J}=\mathcal{R}_{n}$, which means $q=h_{n}$, then Theorem 2 is valid if $s^{3} h_{n}^{2} \log ^{4} h_{n}=o(n)$, $s h_{n}^{2} \log ^{5} h_{n}=o(n), s^{6} \log ^{2} h_{n}=o(n)$ and $n \phi_{n}^{2} \log h_{n}=o(1)$. As discussed in Chang et al. (2018), Proposition 2 holds even if $r$ and $p$ grow at some exponential rate of $n$ with $h_{n} \ll n$. Therefore, the proposed over-identification test can be employed in the case that $r$ and $p$ diverge exponentially.

To show the test is consistent, we assume that under the alternative hypothesis $H_{1}$, there exists some $\varsigma_{n}>0$ that may decay to zero as $n \rightarrow \infty$ such that

$$
\inf _{\theta \in \Theta}\left|E\left\{g_{i}(\theta)\right\}\right|_{\infty} \geq \varsigma_{n} .
$$

Let $\theta_{*}=\arg \inf _{\theta \in \Theta}\left|E\left\{g_{i}(\theta)\right\}\right|_{\infty}$ and $j_{*}=\arg \max _{1 \leq j \leq r}\left|E\left\{g_{i, j}\left(\theta_{*}\right)\right\}\right|$. We impose the following condition.

Condition 7. For $\varsigma_{n}$ specified in (15), it holds that $\left|\bar{g}_{j_{*}}\left(\hat{\theta}_{n}\right)-E\left\{g_{i, j_{*}}\left(\hat{\theta}_{n}\right)\right\}\right|=o_{p}\left(\varsigma_{n}\right)$.

The following theorem states the power of the proposed over-identification test under the alternative hypothesis. 
TheOREM 3. Let (15) and Condition 7 hold under $H_{1}$. Select $\mathcal{J}$ satisfying $\mathcal{J} \supseteq \mathcal{R}_{n}$. If (14) holds and $\varsigma_{n}^{-2} n^{2 / \gamma-1} \log q=o(1)$, then $\operatorname{pr}_{H_{1}}\left(T_{n}>\hat{c v}_{\alpha}\right) \rightarrow 1$ as $n \rightarrow \infty$.

\section{NUMERICAL STUDIES}

\section{5·1. Confidence set estimations}

The methods in $\S 3.1$ are implemented to construct confidence sets in two simulation examples: linear regression model and an analysis of longitudinal data using over-identified estimating equations. We use the function slim in the R package $f$ lare to solve the optimization (8) with the tuning parameter $\tau=0.5\left(n^{-1} \log p\right)^{1 / 2}$, which meets the conditions in our theory. The estimate (2) is used as the initial estimate $\theta^{*}$ in (7) and (8). The smoothly clipped absolute deviation penalty with local quadratic approximation (Fan \& Li, 2001) is employed for both penalty functions $P_{1, \pi}(\cdot)$ and $P_{2, \nu}(\cdot)$ in (2) in all the numerical experiments. The extended Bayesian information criterion (Chen \& Chen, 2008) is applied to determine the tuning parameters $\pi$ and $\nu$ by a two-dimensional grid search. All simulation experiments are repeated for 1,000 times.

Example 1. (Linear regression model) We consider a linear regression model $Y_{i}=Z_{i}^{\mathrm{T}} \theta_{0}+\varepsilon_{i}$, where $\theta_{0}=(1.5,1.2,1,0.9,0.8,0.7,0.6,0.5,0.4,0.3,0, \ldots, 0)^{\mathrm{T}}$ with the first 10 components being nonzero, and $Z_{i} \sim N\left(0, \Sigma_{z}\right)$ with $\Sigma_{z}=\left(\sigma_{z, k l}\right)_{p \times p}$ and $\sigma_{z, k l}=I(k=l)+0.5 I(k \neq l)$. This is a just-identified model. Our method is compared with a few existing alternatives: the desparsified method of van de Geer et al. (2014) implemented by the R package hdi, the estimating equation approach of Neykov et al. (2018) implemented by the R package clime, and the debiased approach of Javanmard \& Montanari (2014) using the code downloaded from the author's website. We consider two settings with $(n, p, r)=(50,100,100)$ and $(100,500,500)$, respectively. Table 1 reports the empirical frequencies of the estimated univariate confidence intervals that cover the truth. At each level, the empirical coverage probabilities are close to the nominal level. We observe that our method has similar coverage accuracy as the de-sparsified method and better coverage accuracy than the the estimating equation approach and the de-biased approach. The average lengths of $95 \%$ confidence intervals are reported in Table 2 . We can see the proposed empirical likelihood-based method outperforms the other methods. The 2-dimensional and 3-dimensional confidence regions constructed from our method are plotted in Figure 1 in the Supplementary Material.

Example 2. (Regression model with repeated measurements) For $m_{i}(i=1, \ldots, n)$ repeated measurements, we consider the model $Y_{i j}=Z_{i j}^{\mathrm{T}} \theta_{0}+\epsilon_{i j}\left(i=1, \ldots, n ; j=1, \ldots, m_{i}\right)$, where $\theta_{0}=(3,1.5,0,0,2,0, \ldots, 0)^{\mathrm{T}}, Z_{i j} \sim N\left(0, \Sigma_{z}\right)$ with $\Sigma_{z}=\left(\sigma_{z, k l}\right)_{p \times p}$ and $\sigma_{z, k l}=0.3^{|k-l|}$, and $\left(\epsilon_{i 1}, \ldots, \epsilon_{i m_{i}}\right)^{\mathrm{T}} \sim N\left(0, \Sigma_{\epsilon}\right)$ with $\Sigma_{\epsilon}=\left(\sigma_{\epsilon, k l}\right)_{p \times p}$ and $\sigma_{\epsilon, k l}=I(k=l)+0.5 I(k \neq l)$. Denote by $Y_{i}=\left(Y_{i 1}, \ldots, Y_{i m_{i}}\right)^{\mathrm{T}}$ and $Z_{i}=\left(Z_{i 1}^{\mathrm{T}}, \ldots, Z_{i m_{i}}^{\mathrm{T}}\right)^{\mathrm{T}}$, respectively, the response variables and the corresponding predictor variables, and write $X_{i}=\left(Y_{i}^{\mathrm{T}}, Z_{i}^{\mathrm{T}}\right)^{\mathrm{T}}$. To incorporate the withinsubject dependence, we apply the estimating function $g\left(X_{i} ; \theta\right)=\left[\left\{Z_{i}^{\mathrm{T}} K_{i}^{-1 / 2} M_{1} K_{i}^{-1 / 2}\left(Y_{i}-\right.\right.\right.$ $\left.\left.\left.Z_{i}^{\mathrm{T}} \theta\right)\right\}^{\mathrm{T}}, \ldots,\left\{Z_{i}^{\mathrm{T}} K_{i}^{-1 / 2} M_{\kappa} K_{i}^{-1 / 2}\left(Y_{i}-Z_{i}^{\mathrm{T}} \theta\right)\right\}^{\mathrm{T}}\right]^{\mathrm{T}}$, as in Qu et al. (2000), where $K_{i} \in \mathbb{R}^{m_{i} \times m_{i}}$ is a diagonal matrix of the conditional variance for subject $i$, and $M_{j}(j=1, \ldots, \kappa)$ are working correlation matrices. We set $m_{i}=3$ and $\kappa=2$ in this smulation with $M_{1}$ being the identity matrix of order 3 and $M_{2}$ being compound symmetry with the diagonal elements of 1 and off-diagonal elements of 0.5 . Since $\kappa=2$, the estimating equations are twice as many as the parameters, so this is an over-identified case with $r=2 p$. For the first five components of the parameters, the empirical frequencies that the estimated confidence intervals cover the truth are reported in Table 3. Similar to Example 1, we see satisfactory performance of the proposed 
Table 1. Empirical frequencies (\%) of the estimated confidence intervals covering the truth in the linear regression example

\begin{tabular}{|c|c|c|c|c|c|c|c|c|c|c|c|c|}
\hline$(n, p, r)$ & Method & Level & $\theta_{1}$ & $\theta_{2}$ & $\theta_{3}$ & $\theta_{4}$ & $\theta_{5}$ & $\theta_{6}$ & $\theta_{7}$ & $\theta_{8}$ & $\theta_{9}$ & $\theta_{10}$ \\
\hline \multirow[t]{12}{*}{$(50,100,100)$} & EL & $90 \%$ & 90.3 & 88.5 & 89.6 & 88.9 & 90.1 & 90.0 & 88.8 & 90.6 & 87.5 & 89.4 \\
\hline & & $95 \%$ & 94.5 & 93.8 & 94.1 & 93.8 & 94.5 & 95.0 & 94.1 & 94.9 & 94.4 & 94.2 \\
\hline & & $99 \%$ & 98.7 & 98.8 & 98.4 & 98.0 & 98.8 & 98.8 & 97.9 & 98.6 & 98.9 & 98.2 \\
\hline & $\mathrm{EE}$ & $90 \%$ & 92.1 & 92.2 & 90.6 & 91.9 & 92.4 & 92.9 & 94.6 & 95.8 & 94.2 & 96.0 \\
\hline & & $95 \%$ & 97.8 & 95.6 & 95.9 & 95.9 & 96.5 & 96.7 & 96.8 & 97.8 & 97.6 & 97.9 \\
\hline & & $99 \%$ & 99.7 & 98.5 & 99.1 & 99.3 & 99.2 & 99.6 & 99.3 & 99.9 & 99.6 & 99.5 \\
\hline & Desparsity & $90 \%$ & 88.9 & 88.8 & 88.1 & 88.0 & 88.2 & 88.2 & 85.5 & 87.6 & 87.4 & 85.5 \\
\hline & & $95 \%$ & 93.6 & 94.0 & 94.1 & 94.3 & 94.7 & 93.5 & 93.0 & 93.8 & 94.5 & 92.3 \\
\hline & & $99 \%$ & 98.7 & 99.4 & 98.9 & 99.1 & 99.3 & 98.6 & 98.8 & 98.5 & 98.5 & 98.5 \\
\hline & Debias & $90 \%$ & 94.3 & 93.0 & 94.0 & 93.9 & 93.2 & 94.3 & 92.6 & 92.7 & 93.5 & 91.5 \\
\hline & & $95 \%$ & 96.3 & 96.5 & 96.5 & 97.3 & 96.5 & 97.4 & 96.5 & 96.0 & 96.7 & 95.8 \\
\hline & & $99 \%$ & 98.5 & 99.5 & 99.5 & 99.6 & 99.3 & 99.6 & 99.3 & 98.7 & 99.8 & 99.0 \\
\hline \multirow[t]{12}{*}{$(100,500,500)$} & EL & $90 \%$ & 88.3 & 88.7 & 89.3 & 89.0 & 88.9 & 89.7 & 88.2 & 88.1 & 89.2 & 89.0 \\
\hline & & $95 \%$ & 93.2 & 94.1 & 94.1 & 93.8 & 93.9 & 93.5 & 94.3 & 93.4 & 94.5 & 94.2 \\
\hline & & $99 \%$ & 98.2 & 98.4 & 98.3 & 98.8 & 98.4 & 98.7 & 98.9 & 98.0 & 98.9 & 98.4 \\
\hline & $\mathrm{EE}$ & $90 \%$ & 93.4 & 92.8 & 92.7 & 91.1 & 91.8 & 91.6 & 94.0 & 94.6 & 95.7 & 95.3 \\
\hline & & $95 \%$ & 97.3 & 96.0 & 96.3 & 95.5 & 95.4 & 95.3 & 96.4 & 97.3 & 98.0 & 97.8 \\
\hline & & $99 \%$ & 99.3 & 98.9 & 98.8 & 99.0 & 99.1 & 99.0 & 99.5 & 99.6 & 99.8 & 99.5 \\
\hline & Desparsity & $90 \%$ & 89.0 & 87.9 & 89.3 & 88.2 & 89.7 & 86.9 & 89.2 & 87.8 & 87.6 & 89.5 \\
\hline & & $95 \%$ & 94.9 & 94.1 & 94.7 & 94.4 & 94.0 & 93.2 & 95.2 & 94.1 & 95.1 & 94.3 \\
\hline & & $99 \%$ & 98.5 & 99.4 & 99.2 & 99.0 & 98.9 & 99.1 & 98.6 & 99.1 & 98.9 & 99.1 \\
\hline & Debias & $90 \%$ & 93.5 & 94.5 & 92.9 & 92.3 & 90.9 & 92.0 & 92.9 & 94.0 & 91.4 & 91.8 \\
\hline & & $95 \%$ & 97.4 & 96.9 & 96.0 & 95.7 & 95.7 & 95.5 & 96.4 & 96.7 & 95.5 & 96.0 \\
\hline & & $99 \%$ & 99.6 & 99.1 & 99.1 & 99.1 & 99.0 & 99.1 & 99.7 & 99.3 & 99.2 & 99.1 \\
\hline
\end{tabular}

EL: the proposed method; EE: Neykov et al. (2018)'s method; Desparsity: van de Geer et al. (2014)'s method; Debias: Javanmard \& Montanari (2014)'s method.

Table 2. Average lengths of the $95 \%$ confidence intervals in the linear regression example with $(n, p, q)=(50,100,100)$

$\begin{array}{ccccccccccc}\text { Method } & \theta_{1} & \theta_{2} & \theta_{3} & \theta_{4} & \theta_{5} & \theta_{6} & \theta_{7} & \theta_{8} & \theta_{9} & \theta_{10} \\ \text { EL } & 0.815 & 0.769 & 0.771 & 0.781 & 0.758 & 0.749 & 0.770 & 0.760 & 0.789 & 0.782 \\ \text { EE } & 0.905 & 0.884 & 0.887 & 0.862 & 0.889 & 0.867 & 0.857 & 0.867 & 0.854 & 0.869 \\ \text { Desparsity } & 0.837 & 0.841 & 0.852 & 0.856 & 0.867 & 0.846 & 0.841 & 0.851 & 0.847 & 0.864 \\ \text { Debias } & 0.840 & 0.841 & 0.845 & 0.849 & 0.859 & 0.840 & 0.842 & 0.851 & 0.849 & 0.867\end{array}$

method in the over-identified case. The 2-dimensional and 3-dimensional empirical likelihood based confidence regions are plotted in Figure 2 in the Supplementary Material.

The sequential procedure described at the end of $\S 3 \cdot 1$ is also implemented by finding two estimating equations for each individual component in this over-identified example. We found similar coverage accuracy for such a method. To see its advantage, Table 4 compares the lengths of $95 \%$ confidence intervals using one estimating equation versus two estimating equations. It can be seen that the confidence intervals using two estimating equations are about $11 \%$ shorter than those using only one estimating equation, which shows a potential advantage of our method since more information can be retained through the over-identification of estimating functions.

\subsection{Over-identification test}

To assess the over-identification test in $\S 3 \cdot 2$, we consider the mean of a normal distributed random vector $X=\left(X_{1}, \ldots, X_{p}\right)^{\mathrm{T}}$, where only the first component $X_{1}$ has a nonzero mean of 5 and the rest components all have mean zero. The first $p$ estimating functions are simply from 
Table 3. Empirical frequencies (\%) of the empirical likelihood based confidence intervals covering the truth in the repeated measurements example

$\begin{array}{ccccccc}(n, p, r) & \text { Level } & \theta_{1} & \theta_{2} & \theta_{3} & \theta_{4} & \theta_{5} \\ (50,100,200) & 90 \% & 87.3 & 88.3 & 89.6 & 89.9 & 88.9 \\ & 95 \% & 93.4 & 93.6 & 94.9 & 94.5 & 93.8 \\ (100,200,400) & 99 \% & 97.5 & 98.0 & 98.8 & 98.4 & 98.2 \\ & 90 \% & 89.3 & 89.1 & 92.5 & 92.5 & 88.9 \\ & 95 \% & 93.8 & 94.5 & 96.4 & 96.2 & 94.8 \\ & 99 \% & 98.0 & 98.9 & 99.2 & 98.9 & 98.6\end{array}$

Table 4. Comparison of lengths of the $95 \%$ empirical likelihood based confidence intervals in the repeated measurements example with $(n, p, r)=(50,100,200)$

$\begin{array}{cccccc}\text { Method } & \theta_{1} & \theta_{2} & \theta_{3} & \theta_{4} & \theta_{5} \\ \text { One estimating equation } & 0.325 & 0.329 & 0.323 & 0.322 & 0.323 \\ \text { Two estimating equations } & 0.289 & 0.293 & 0.285 & 0.288 & 0.285\end{array}$

the components of $g(X ; \theta)=X-\theta$ with $\theta_{0}=(5,0, \ldots, 0)^{\mathrm{T}}$. In addition, we impose an extra moment restriction $g_{p+1}(X ; \theta)=X_{1}^{2}-\theta_{1}^{2}-25$ where $\theta_{1}$ is the first component of $\theta$. In this setting, the number of estimating equations is $r=p+1$. We consider the following two cases:

Case 1. The covariance matrix $\Sigma=\left(\sigma_{i j}\right)_{p \times p}$ is compound symmetry with diagonal $\sigma_{11}=5^{2}$ and $\sigma_{i i}=1$ for all $i \neq 1$. All off-diagonal elements $\sigma_{i j}=0.3$ for $i \neq j$;

Case 2. The covariance matrix $\Sigma=\left(\sigma_{i j}\right)_{p \times p}$ is compound symmetry with diagonal $\sigma_{11}=5^{2} \times a$ with $a<1$ and $\sigma_{i i}=1$ for all $i \neq 1$. All off-diagonal elements $\sigma_{i j}=0.3$ for $i \neq j$.

Clearly, the moment conditions are correctly specified in Case 1 but not in Case 2. We conduct the experiments for a few settings of $(n, p, r)$ in this example. We apply (13) to obtain the critical value of the test. Further, we compare the performances of the test by using two different choices of $\mathcal{J}$ in (9). The first one, referred to as Method 1 , uses the set $\mathcal{R}_{n}$ of estimating functions selected by (2). The other one, referred to as Method 2, simply uses $\mathcal{J}$ containing all estimating functions. We report in Table 5 the empirical percentages rejecting $H_{0}$ at $\alpha=0.05$ level. In Case 1 , we expect that the rate to be close to 0.05 , which indeed the case for our advocated Method 1 for choosing $\mathcal{R}_{n}$ as $\mathcal{J}$. Method 2 works well when the dimension is low, but it get much worse with $p$ and $r$ getting close to $n$. In Case 2, the closer the rate is to 1 , the better the power is for the testing procedure. We tried three settings with $a=0.7,0.5$ and 0.3 respectively, where smaller value in $a$ can be viewed as more severe violation of $H_{0}$. One can clearly see that the advocated method works quite well in terms of providing a more powerful test with the right choice of estimating functions. The power improves consistently for more severe violation of the null hypothesis. As for the Method 2, it works well when the $p$ and $r$ are small, but it becomes powerless in moderate high-dimensional cases, which is consistent with our discussions in $\S 3 \cdot 2$.

\section{5·3. Multi-level longitudinal study of physical activity among girls}

We analyze a data set from a longitudinal study of physical activities among girls from adolescence into young adulthood. The main goal of this study is to identify individual, social, and environmental factors associated with moderate to vigorous physical activity among those girls over time using a multi-level approach. An initial cohort of 730 girls were randomly recruited in Maryland in 2006 and 428 girls had complete assessments at all three study periods in 2006 $(n=730), 2009(n=589)$, and $2015(n=460)$ at ages 14,17 , and 23. The response variable, moderate to vigorous physical activity minutes, were assessed from accelerometers and over 800 
Table 5. Empirical percentages of rejecting $H_{0}$ in the model specification test example. Case 1 corresponds to a correct model specification and Case 2 corresponds to a model misspecification; Method 1 uses the selected set of estimating functions by $\mathcal{R}_{n}$, and Method 2 uses all the estimating functions

\begin{tabular}{|c|c|c|c|c|}
\hline & $\sigma_{11}$ & $(n, p)$ & Method 1 & Method 2 \\
\hline \multirow[t]{5}{*}{ Case 1} & $5^{2}$ & $(50,1)$ & 0.056 & 0.056 \\
\hline & & $(50,10)$ & 0.061 & 0.061 \\
\hline & & $(50,50)$ & 0.061 & 0.002 \\
\hline & & $(50,100)$ & 0.058 & 0.002 \\
\hline & & $(100,100)$ & 0.047 & 0.002 \\
\hline \multirow[t]{15}{*}{ Case 2} & $5^{2} \times 0.7$ & $(50,1)$ & 0.492 & 0.492 \\
\hline & & $(50,10)$ & 0.521 & 0.521 \\
\hline & & $(50,50)$ & 0.580 & 0.082 \\
\hline & & $(50,100)$ & 0.601 & 0.054 \\
\hline & & $(100,100)$ & 0.738 & 0.286 \\
\hline & $5^{2} \times 0.5$ & $(50,1)$ & 0.915 & 0.915 \\
\hline & & $(50,10)$ & 0.911 & 0.911 \\
\hline & & $(50,50)$ & 0.883 & 0.143 \\
\hline & & $(50,100)$ & 0.890 & 0.257 \\
\hline & & $(100,100)$ & 0.994 & 0.381 \\
\hline & $5^{2} \times 0.3$ & $(50,1)$ & 1.000 & 1.000 \\
\hline & & $(50,10)$ & 1.000 & 1.000 \\
\hline & & $(50,50)$ & 0.998 & 0.167 \\
\hline & & $(50,100)$ & 1.000 & 0.743 \\
\hline & & $(100,100)$ & 1.000 & 0.294 \\
\hline
\end{tabular}

variables were collected, including: (i) demographic and psychosocial information (individualand social-level variables); (ii) height, weight, and triceps skinfold to assess body composition; and (iii) geographical information systems and self-report for neighborhood-level variables.

In this example, we consider an over-identified model specification with $r>p$; see the longitudinal data example in $\S 5 \cdot 1$. The same estimating equations and basis matrices $M_{1}$ and $M_{2}$ of size $3 \times 3$ as in $\S 5 \cdot 1$ are used. Eight predictor variables out of thirty-four screened variables were selected in the model for the logarithm of response (Table 6). The second column of Table 6 provides the regression coefficients together with the $95 \%$ component-wise confidence intervals estimated by the approach in $\S 3.1$ using the over-identified estimating equations. We see that none of the $95 \%$ confidence intervals contain 0 , showing that all the selected variables are statistically significant in the model. We applied the over-identification test in $\S 3 \cdot 2$, and found no significant statistical evidence against the model specification with over-identification.

In the selected model, the first variable TAAG is an ordinal variable indicating the time of study when data were collected. As expected, physical activities decreased significantly over time among young females. The variable self-management strategies, an aggregated variable of 8 questionnaire items, and social support from friends, a sum of 3 questionnaire items, are positively correlated with the response. parents' education and number of parks with 1 mile distance from home have positive impact on physical activities. On the other hand, BMI and being a smoker are negatively correlated with physical activities. Our findings are consistent with the previous results (Young et al., 2014; Grant et al., 2015; Young et al., 2018).

As for comparisons, we apply an alternative approach using a linear regression model. The third column of Table 6 reports the component-wise point estimates and confidence intervals for the eight selected variables. All the confidence intervals in this approach are wider than those from the over-identified estimating equations; the ratios of the interval lengths are reported in 
Table 6. The estimated regression coefficients and 95\% confidence intervals for the selected variables associated with MVPA over time using penalized empirical likelihood, as compared to linear regression. The column C.I. Ratio lists the ratio of the $95 \%$ confidence intervals constructed from over-identified estimating functions and the linear models

$\begin{array}{lllc}\text { Variable } & \text { Repeated } & \text { Linear Reg. } & \text { C.I. Ratio } \\ \text { TAAG (time) } & -0.280(-0.310,-0.210) & -0.297(-0.356,-0.237) & 0.840 \\ \text { Body mass index } & -0.056(-0.136,-0.016) & -0.098(-0.163,-0.041) & 0.984 \\ \text { Self-management strategies } & 0.072(0.052,0.172) & 0.126(0.065,0.186) & 0.992 \\ \text { Social support from friends } & 0.118(0.048,0.148) & 0.079(0.023,0.135) & 0.890 \\ \text { Smoker } & -0.102(-0.132,-0.022) & -0.044(-0.100,0.011) & 0.991 \\ \text { Father's education } & 0.059(0.029,0.139) & 0.087(0.023,0.151) & 0.859 \\ \text { Mother's education } & 0.067(0.037,0.147) & 0.073(0.010,0.137) & 0.862 \\ \text { Number of parks within 1 mile } & 0.088(0.058,0.178) & 0.126(0.061,0.182) & 0.992\end{array}$

the fourth column of Table 6. In particular, the variable smoker is significant when applying the over-identified approach, but becomes insignificant if ignoring the within-subject dependence.

The two-dimensional confidence regions for TAAG (i.e., time) versus other covariates are in Figure 3 in the Supplementary Material. The constructed confidence regions are not symmetric at the estimate, reflecting the merit that the empirical likelihood-based confidence region is data oriented and free of shape constraint.

\section{DISCUSSION}

It would be interesting to extend high-dimensional statistical inference to a setting with some unknown functional-valued parameters. A possible strategy is to apply the sieve method (Ai \& Chen, 2003) to approximate the functional-valued parameters with some linear combinations of diverging number of given basis functions. Then the estimation of unknown functionalvalued parameters is transferred to the estimation of the coefficients in the sieve approximation; and the frameworks of Chang et al. (2018) and this paper apply. Nevertheless, accommodating functional-valued parameters will introduce foundational changes in the settings. New developments in both theory and methods are beyond the scope of this study. We plan to carefully investigate the problem in a future project.

\section{ACKNOWLEDGEMENT}

We are grateful to the editor, associate editor and referees for their constructive comments and helpful suggestions. We also thank Matey Neykov for sharing the R code. Chang was supported in part by the Fundamental Research Funds for the Central Universities of China, the National Natural Science Foundation of China, the Fok Ying-Tong Education Foundation, and the Center of Statistical Research, the Joint Lab of Data Science and Business Intelligence at Southwestern University of Finance and Economics. Tang acknowledges support from the U.S. National Science Foundation. Wu's research was partly supported by U.S. National Institutes of Health and U.S. National Science Foundation.

\section{SUPPLEMENTARY MATERIAL}

Supplementary material available at Biometrika online includes all the technical proofs, figures of the empirical likelihood based confidence regions in $\S 5$, and an extra simulation example. 


\section{APPENDIX}

Write $\theta_{0}=\left(\theta_{0,1}, \ldots, \theta_{0, p}\right)^{\mathrm{T}}$. Recall $\mathcal{S}=\left\{1 \leq k \leq p: \theta_{0, k} \neq 0\right\}$. To investigate the properties of the penalized empirical likelihood estimator $\hat{\theta}_{\mathrm{PEL}}$ defined as (2), we need the following technical conditions.

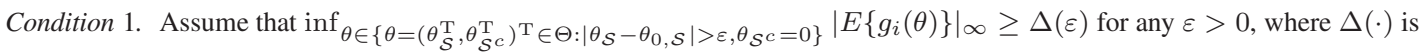
a function satisfying $\liminf _{\varepsilon \rightarrow 0^{+}} \varepsilon^{-\beta} \Delta(\varepsilon) \geq K_{1}$ for some uniform constants $K_{1}>0$ and $\beta \in(0,1]$.

For some $C_{*} \in(0,1)$, define $\mathcal{M}_{\theta}=\left\{1 \leq j \leq r:\left|\bar{g}_{j}(\theta)\right| \geq C_{*} \nu \rho_{2}^{\prime}\left(0^{+}\right)\right\}$for any $\theta \in \Theta$. Let $b_{n}=\max \left\{a_{n}, \nu^{2}\right\}$ with $a_{n}=\sum_{k=1}^{p} P_{1, \pi}\left(\left|\theta_{0, k}\right|\right)$, and

$$
l_{n}=\max _{\theta \in\left\{\theta=\left(\theta_{\mathcal{S}}^{\mathrm{T}}, \theta_{\mathcal{S}^{c}}^{\mathrm{T}}\right)^{\mathrm{T}} \in \Theta:\left|\theta_{\mathcal{S}}-\theta_{0, \mathcal{S}}\right|_{\infty} \leq c_{n}, \theta_{\left.\mathcal{S}^{c}=0\right\}}\right.}\left|\mathcal{M}_{\theta}\right|
$$

for some $c_{n} \rightarrow 0$ satisfying $b_{n}^{1 /(2 \beta)} c_{n}^{-1} \rightarrow 0$ with $\beta$ specified in Condition A1. We assume $l_{n} \geq s$ with $s=|\mathcal{S}|$.

Condition 2. For any $X$ and $j=1, \ldots, r, g_{j}(X ; \theta)$ is continuously differentiable with respect to $\theta$. It holds that $\max _{1 \leq j \leq r} \max _{k \notin \mathcal{S}} E\left\{\sup _{\theta \in \Theta}\left|\partial g_{i, j}(\theta) / \partial \theta_{k}\right|\right\} \leq K_{2} \quad$ for $\quad$ some $\quad$ uniform constant $K_{2}>0$, and $\sup _{\theta \in \Theta} \max _{1 \leq j \leq r} \max _{k \notin \mathcal{S}} n^{-1} \sum_{i=1}^{n}\left|\partial g_{i, j}(\theta) / \partial \theta_{k}\right|=O_{p}(1)$.

Condition 3. It holds that $\max _{1 \leq j \leq r} E\left\{\sup _{\theta \in \Theta}\left|g_{i, j}(\theta)\right|^{\gamma}\right\} \leq K_{3}$ for some uniform constants $K_{3}>0$ and $\gamma>4$.

Condition 4. Let $V_{\mathcal{F}}\left(\theta_{0}\right)=E\left\{g_{i, \mathcal{F}}(\theta)^{\otimes 2}\right\}$ for any $\mathcal{F} \subset\{1, \ldots, r\}$. There exist uniform constants $0<K_{4}<K_{5}$ such that $K_{4}<\lambda_{\min }\left\{V_{\mathcal{F}}\left(\theta_{0}\right)\right\}<\lambda_{\max }\left\{V_{\mathcal{F}}\left(\theta_{0}\right)\right\}<K_{5}$ for any $\mathcal{F}$ with $|\mathcal{F}| \leq l_{n}$, where $l_{n}$ is defined as (R.1).

Condition 5. It holds that $\sup _{\theta \in \Theta} \max _{1 \leq j \leq r} \max _{1 \leq k \leq p} n^{-1} \sum_{i=1}^{n}\left|\partial g_{i, j}(\theta) / \partial \theta_{k}\right|=O_{p}(1) \quad$ and $\sup _{\theta \in \Theta} \max _{1 \leq j \leq r} n^{-1} \sum_{i=1}^{n}\left|g_{i, j}(\theta)\right|^{4}=O_{p}(1)$.

Condition 6. For $c_{n}$ specified in (R.1), it holds that $\max _{k \in \mathcal{S}} \sup _{0<t<\left|\theta_{0, k}\right|+c_{n}} P_{1, \pi}^{\prime}(t)=O\left(\chi_{n}\right)$ for some $\chi_{n} \rightarrow 0$.

Conditions A1-A6 are the simplified version of Conditions 1-6 in Chang et al. (2018). We refer to Chang et al. (2018) for the detailed discussion of their validity. With the additional assumption $b_{n}=o\left(\min _{k \in \mathcal{S}}\left|\theta_{0, k}\right|^{2 \beta}\right)$ that the signal strength of the nonzero components of $\theta_{0}$ does not diminish to zero too fast, Condition A6 can be replaced by

$$
\max _{k \in \mathcal{S}} \sup _{c\left|\theta_{0, k}\right|<t<c^{-1}\left|\theta_{0, k}\right|} P_{1, \pi}^{\prime}(t)=O\left(\chi_{n}\right) .
$$

For those asymptotically unbiased penalties like the smoothly clipped absolute deviation penalty (Fan \& Li, 2001) and the minimax concave penalty (Zhang, 2010), $\chi_{n}=0$ in (R.2) for $n$ sufficiently large if $b_{n}=o\left(\min _{k \in \mathcal{S}}\left|\theta_{0, k}\right|^{2 \beta}\right)$. Define $\kappa_{n}=$ $\max \left\{l_{n}^{1 / 2} n^{-1 / 2}, s^{1 / 2} \chi_{n}^{1 / 2} b_{n}^{1 /(4 \beta)}\right\}$. Based on Conditions A1-A6, Proposition 1 holds provided that the following restrictions are satisfied:

$$
\begin{gathered}
\log r=o\left(n^{1 / 3}\right), s^{2} l_{n} b_{n}^{1 / \beta}=o(1), l_{n}^{2} n^{-1} \log r=o(1), \max \left\{b_{n}, l_{n} \kappa_{n}^{2}\right\}=o\left(n^{-2 / \gamma}\right), \\
l_{n}^{1 / 2} \kappa_{n}=o(\nu) \text { and } l_{n}^{1 / 2} \max \left\{l_{n} \nu, s^{1 / 2} \chi_{n}^{1 / 2} b_{n}^{1 /(4 \beta)}\right\}=o(\pi) .
\end{gathered}
$$

The convergence rate $\alpha_{n}$ specified in Proposition 1 equals to $b_{n}^{1 /(2 \beta)}$. If $b_{n}=o\left(\min _{k \in \mathcal{S}}\left|\theta_{0, k}\right|^{2 \beta}\right)$ and $P_{1, \pi}(\cdot)$ is selected as the asymptotically unbiased penalty, $\chi_{n}$ specified in (R.2) can be selected as 0. Since $a_{n}=O(s \pi)$, restrictions (R.3) in this scenario can be simplified as $\log r=o\left(n^{1 / 3}\right), l_{n}=o\left(\min \left\{n^{1 / 2}(\log r)^{-1 / 2}, n^{1 / 2-1 / \gamma}\right\}\right)$, and the tuning parameters $\nu$ and $\pi$ satisfy $l_{n} n^{-1 / 2}=o(\nu), \nu=o\left(\min \left\{s^{-\beta} l_{n}^{-\beta / 2}, n^{-1 / \gamma}\right\}\right), l_{n}^{3 / 2} \nu=o(\pi)$ and $\pi=o\left(\min \left\{s^{-2 \beta-1} l_{n}^{-\beta}, s^{-1} n^{-2 / \gamma}\right\}\right)$. If $\log r=o\left(n^{1 / 3}\right)$ and $l_{n}=o\left(\min \left\{n^{(\gamma-4) /(5 \gamma)} s^{-2 / 5}, n^{1 /(2 \beta+5)} s^{-(4 \beta+2) /(2 \beta+5)}\right\}\right)$, there exist suitable selections of $\nu$ and $\pi$ satisfying these conditions. Furthermore, we need two additional conditions listed below to construct Proposition 2.

Condition 7. For any $X$ and $j=1, \ldots, p, g_{j}(X ; \theta)$ is twice continuously differentiable with respect to $\theta$, and $\sup _{\theta \in \Theta} \max _{1 \leq j \leq r} \max _{k_{1}, k_{2} \in \mathcal{S}} n^{-1} \sum_{i=1}^{n}\left|\partial^{2} g_{i, j}(\theta) / \partial \theta_{k_{1}} \partial \theta_{k_{2}}\right|^{2}=O_{p}(1)$.

Condition 8. Let $Q_{\mathcal{F}}=\left(\left[E\left\{\nabla_{\theta_{\mathcal{S}}} g_{i, \mathcal{F}}\left(\theta_{0}\right)\right\}\right]^{\mathrm{T}}\right)^{\otimes 2}$ for any $\mathcal{F} \subset\{1, \ldots, r\}$. There exist uniform constants $0<K_{6}<K_{7}$ such that $K_{6}<\lambda_{\min }\left(Q_{\mathcal{F}}\right) \leq \lambda_{\max }\left(Q_{\mathcal{F}}\right)<K_{7}$ for any $\mathcal{F}$ with $s \leq|\mathcal{F}| \leq l_{n}$, where $l_{n}$ is defined as (R.1).

Based on Conditions A1-A8, Proposition 2 holds provided that the following restrictions are satisfied:

$$
\begin{gathered}
\log r=o\left(n^{1 / 3}\right), b_{n}=o\left(n^{-2 / \gamma}\right), n s \chi_{n}^{2}=o(1), n l_{n} \kappa_{n}^{4} \max \left\{s, n^{2 / \gamma}\right\}=o(1), \\
l_{n}^{2}(\log r) \max \left\{s^{3} b_{n}^{1 / \beta}, l_{n} n^{-1} \log r\right\}=o(1), n l_{n} s^{2} \max \left\{l_{n}^{2} \nu^{4}, s^{2} \chi_{n}^{2} b_{n}^{1 / \beta}\right\}=o(1), \\
l_{n}^{1 / 2} \kappa_{n}=o(\nu) \text { and } l_{n}^{1 / 2} \max \left\{l_{n} \nu, s^{1 / 2} \chi_{n}^{1 / 2} b_{n}^{1 /(4 \beta)}\right\}=o(\pi) .
\end{gathered}
$$

Notice that $a_{n}=O(s \pi)$. Under the reasonable case $\chi_{n}=0$ and $l_{n} \sim s$, restrictions (R.4) hold provided that $\log r=$ $o\left(n^{1 / 3}\right), s=o\left(\min \left\{n^{1 / 9}, n^{1 /(10 \beta+7)}(\log r)^{-2 \beta /(10 \beta+7)}, n^{(\gamma-4) /(7 \gamma)}\right\}\right)$, and the tuning parameters $\nu$ and $\pi$ satisfy conditions $\pi=o\left(\min \left\{n^{-2 / \gamma} s^{-1}, s^{-5 \beta-1}(\log r)^{-\beta}\right\}\right), \nu=o\left(\min \left\{n^{-1 / \gamma}, s^{-5 \beta / 2}(\log r)^{-\beta / 2}, n^{-1 / 4} s^{-5 / 4}\right\}\right), s^{3 / 2} \nu=o(\pi)$ 
and $s n^{-1 / 2}=o(\nu)$. Due to $s=o\left(\min \left\{n^{1 / 9}, n^{1 /(10 \beta+7)}(\log r)^{-2 \beta /(10 \beta+7)}, n^{(\gamma-4) /(7 \gamma)}\right\}\right)$, there exist suitable selections of $\nu$ and $\pi$ satisfying these conditions.

\section{REFERENCES}

Ai, C. \& Chen, X. (2003). Efficient estimation of models with conditional moment restrictions containing unknown functions. Econometrica, 71, 1795-1843.

Belloni, A. \& Chernozhukov, V. (2013). Least squares after model selection in high-dimensional sparse models. Bernoulli, 19, 521-547.

Bickel, P., Ritov, Y. \& Tsybakov, A. B. (2009). Simultaneous analysis of Lasso and Dantzig selector. Ann. Statist., 37 , 1705-1732.

Bravo, F., Escanciano, J. C. \& Van Keilegom, I. (2019). Two-step semiparametric empirical likelihood inference. Ann. Statist., in press.

Bühlmann, P. \& van de Geer, S. (2011). Statistics for High-dimensional Data: Methods, Theory and Applications. New York: Springer.

Candès, E. \& Tao, T. (2007). The Dantzig selector: Statistical estimation when $p$ is much larger than $n$. Ann. Statist., $35,2313-2351$.

Chang, J., Chen, S. X. \& Chen, X. (2015). High dimensional generalized empirical likelihood for moment restrictions with dependent data. J. Econometrics, 185, 283-304.

Chang, J., Tang, C. Y. \& Wu, T. T. (2018). A new scope of penalized empirical likelihood with high-dimensional estimating equations. Ann. Statist., 46, 3185-3216.

Chang, J., Tang, C. Y. \& Wu, Y. (2013). Marginal empirical likelihood and sure independence feature screening. Ann. Statist., 41, 2132-2148.

Chang, J., Tang, C. Y. \& Wu, Y. (2016). Local independence feature screening for nonparametric and semiparametric models by marginal empirical likelihood. Ann. Statist., 44, 515-539.

Chang, J., Zheng, C., Zhou, W.-X. \& Zhou, W. (2017). Simulation-based hypothesis testing of high dimensional means under covariance heterogeneity. Biometrics, 73, 1300-1310.

Chen, J. \& Chen, Z. (2008). Extended Bayesian information criterion for model selection with large model space. Biometrika, 95, 759-771.

Chen, S. X. \& Cui, H. J. (2006). On Bartlett correction of empirical likelihood in the presence of nuisance parameters. Biometrika, 93, 215-220.

Chen, S. X. \& Cui, H. J. (2007). On the second-order properties of empirical likelihood with moment restrictions. $J$. Econometrics, 141, 492-516.

Chen, S. X., Peng, L. \& Qin, Y. (2009). Effects of data dimension on empirical likelihood. Bimetrika, 96, 711-722.

Chernozhukov, V., Chetverikov, D. \& Kato, K. (2017). Central limit theorems and bootstrap in high dimensions. Ann. Prob., 45, 2309-2352.

Fan, J. \& Li, R. (2001). Variable selection via nonconcave penalized likelihood and its oracle properties. J. Amer. Statist. Assoc., 96, 1348-1360.

Fan, J. \& Lv, J. (2010). A selective overview of variable selection in high dimensional feature space. Statist. Sinica, $20,101-148$.

Grant, E. M., Young, D. R. \& Wu, T. T. (2015). Predictors for physical activity in adolescent girls using statistical shrinkage techniques for hierarchical longitudinal mixed effects models. PLOS ONE, 10, e0125431.

Hall, P. \& La Scala, B. (1990). Methodology and algorithms of empirical likelihood. Internat. Statist. Rev., 58, 109127.

Hansen, L. P. (1982). Large sample properties of generalized method of moments estimators. Econometrica, 50, $1029-1054$.

Hansen, L. P. \& Singleton, K. J. (1982). Generalized instrumental variables estimation of nonlinear rational expectations models. Econometrica, 50, 1269-1286.

Hastie, T., Tibshirani, R. \& Wainwright, M. (2015). Statistical Learning with Sparsity: The Lasso and Generalizations. Taylor \& Francis Inc.

Hjort, N. L., McKeague, I. \& Van Keilegom, I. (2009). Extending the scope of empirical likelihood. Ann. Statist., 37, 1079-1111.

Javanmard, A. \& Montanari, A. (2014). Confidence intervals and hypothesis testing for high-dimensional regression. J. Mach. Learn. Res., 15, 2869-2909.

Lazar, N. A. \& Mykland, P. A. (1999). Empirical likelihood in the presence of nuisance parameters. Biometrika, 86, 203-211.

Lee, J. D., Sun, D. L., Sun, Y. \& Taylor, J. E. (2016). Exact post-selection inference, with application to the lasso. Ann. Statist., 44, 907-927.

Leng, C. \& Tang, C. Y. (2012). Penalized empirical likelihood and growing dimensional general estimating equations. Biometrika, 99, 703-716. 
Neykov, M., Ning, Y., Liu, J. S. \& Liu, H. (2018). A unified theory of confidence regions and testing for high dimensional estimating equations. Stat. Sci., 33, 427-443.

Ning, Y. \& Liu, H. (2017). A general theory of hypothesis tests and confidence regions for sparse high dimensional models. Ann. Statist., 45, 158-195.

Owen, A. B. (2001). Empirical Likelihood. New York: Chapman and Hall/CRC.

Petrov, V. V. (1995). Limit Theorems of Probability Theory: Sequences of Independent Random Variables. Oxford University Press.

Qin, J. \& Lawless, J. (1994). Empirical likelihood and general estimating equations. Ann. Statist., 22, 300-325.

Qin, J. \& Lawless, J. (1995). Estimating equations, empirical likelihood and constraints on parameter. Can. J. Stat., $23,145-159$.

Qu, A., Lindsay, B. G. \& Li, B. (2000). Improving estimating equations using quadratic inference functions. Biometrika, 87, 823-836.

Sargan, J. D. (1958). The estimation of economic relationships using instrumental variables. Econometrica, 26, 393415.

Tibshirani, R. J., Taylor, J., Lockhart, R. \& Tibshirani, R. (2016). Exact post-selection inference for sequential regression procedures. J. Amer. Statist. Assoc., 111, 600-620.

van de Geer, S., Bülmann, P., Ritov, Y. \& Dezeure, R. (2014). On asymptotically optimal confidence regions and tests for high-dimensional models. Ann. Statist., 42, 1166-1202.

Young, D., Saksvig, B. I., Wu, T. T., Zook, K., Li, X., Champaloux, S., Grieser, M., Lee, S. \& Treuth, M. S. (2014). Multilevel correlates of physical activity for early, mid, and late adolescent girls. J. Phys. Act. Health, 11, 950-960.

Young, D. R., Cohen, D., Koebnick, C., Mohan, Y., Saksvig, B. I., Sidell, M. \& Wu, T. T. (2018). Longitudinal associations of physical activity among females from adolescence to young adulthood. J. Adolesc. Health, 63, 466-473.

Zhang, C.-H. (2010). Nearly unbiased variable selection under minimax concave penalty. Ann. Statist., 38, 894-942.

Zhang, C.-H. \& Zhang, S. S. (2013). Confidence intervals for low dimensional parameters in high dimensional linear models. J. R. Stat. Soc. Ser. B. Stat. Methodol., 76, 217-242.

[Received 11 April2019. Revised 1 November2019] 